\title{
Chapter 10 \\ The Butterflies and Skippers (Lepidoptera: \\ Papilionoidea) of Angola: An Updated Checklist
}

\author{
Luís F. Mendes, A. Bivar-de-Sousa, and Mark C. Williams
}

\begin{abstract}
Presently, 792 species/subspecies of butterflies and skippers (Lepidoptera: Papilionoidea) are known from Angola, a country with a rich diversity of habitats, but where extensive areas remain unsurveyed and where systematic collecting programmes have not been undertaken. Only three species were known from Angola in 1820. From the beginning of the twenty-first century, many new species have been described and more than 220 faunistic novelties have been assigned. As a whole, of the 792 taxa now listed for Angola, 57 species/subspecies are endemic and almost the same number are known to be near-endemics, shared by Angola and by one or another neighbouring country. The Nymphalidae are the most diverse family. The Lycaenidae and Papilionidae have the highest levels of endemism. A revised checklist with taxonomic and ecological notes is presented and the development of knowledge of the superfamily over time in Angola is analysed.
\end{abstract}

Keywords Africa $\cdot$ Conservation $\cdot$ Ecology $\cdot$ Endemism $\cdot$ Taxonomy

\footnotetext{
L. F. Mendes ( $\square)$

Museu Nacional de História Natural e da Ciência, Universidade de Lisboa, Lisboa, Portugal CIBIO, Centro de Investigação em Biodiversidade e Recursos Genéticos, Vairão, Portugal e-mail: luisfmendes22@gmail.com
}

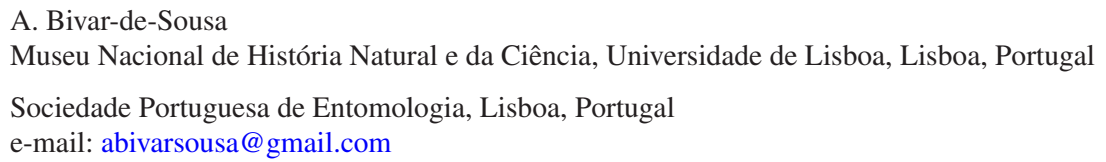




\section{Introduction}

Angola is a large country of $1,246,700 \mathrm{~km}^{2}$, notable for its great diversity of physiography, climates, habitats and resultant biodiversity). The country includes seven biomes and 15 ecoregions, ranging from equatorial rainforests of the northwest (Cabinda) and along the northern border with the Democratic Republic of Congo, through the moist miombo woodlands and savannas of the central plateaus, to the dry forests and woodlands of the southeast, and to the arid shrublands and Namib Desert of the southwest. Isolated forests with Congolian affinities are found along the Angolan Escarpment, and similar remnant patches of Afromontane forests are found on some of the highest mountains such as Mount Moco and Mount Namba.

Despite the fact that at the beginning of the nineteenth century only a few species of butterflies and skippers (Insecta: Lepidoptera: Papilionoidea) were recorded from Angola, today a large number of taxa (at least 792 species and subspecies: Fig. 10.1, Table 10.1 and Appendix) are known to occur in the country. However, extensive areas are still poorly surveyed for butterflies, or have not been surveyed at all (Fig. 10.2). This applies in particular to the southern provinces of Namibe, Cunene and Cuando Cubango and the northwestern province of Zaire as well as most of southern Moxico. Furthermore, the Baixa de Cassanje (Malanje), separated from surrounding areas by steep escarpments, appears to have distinctive vegetation and may produce some interesting butterflies. Although most of the localities where but-

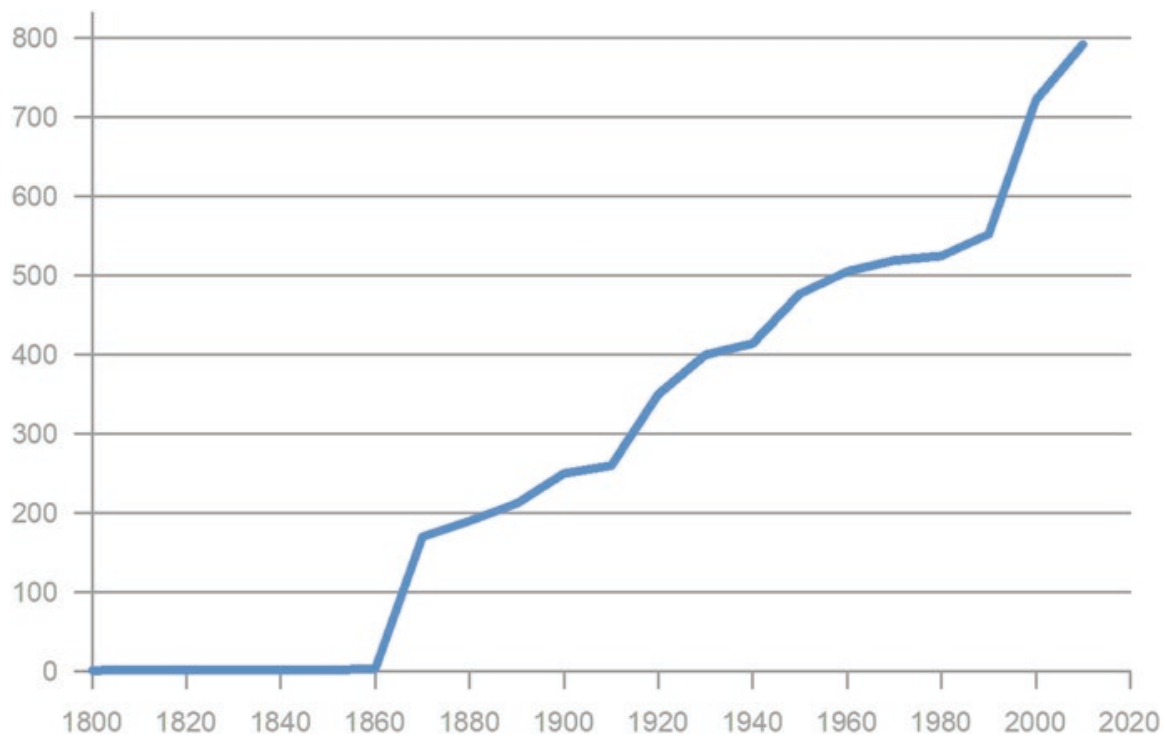

Fig. 10.1 Cumulative number of species/subspecies of Papilionoidea reported from Angola from 1801-1819 (first records) to the recent decade - 2011-2017 - according to Appendix. For practical reasons, species which first reference to the country was untraceable (marked in Appendix with a A) were included in the decade 2001-2010; species that are now assigned as faunistic novelties to Angola (marked in Appendix with a

-) are included in the last decade (2011-2017) 
Table 10.1 Number of species of Papilionoidea families and subfamilies known to occur in the Afrotropical Region and Angola (with \% of Afrotropical species present in the country), and number of species endemic to Angola (with \% of endemism shown)

\begin{tabular}{|c|c|c|c|}
\hline Family & Afrotropical & Angola & Endemism \\
\hline Subfamily & $\mathrm{N}^{\circ}$ & $\mathrm{N}^{\circ} \mid \%$ & $\mathrm{~N} \mid \%$ \\
\hline HESPERIIDAE & 618 & $134 \mid 22$ & $5 \mid 3.7$ \\
\hline Coeliadinae & 21 & $7 \mid 33$ & \\
\hline Pyrginae & 216 & $48 \mid 22$ & \\
\hline Heteropterinae & 27 & $5 \mid 19$ & \\
\hline Hesperiinae & 334 & $74 \mid 22$ & \\
\hline PAPILIONIDAE & 101 & $33 \mid 33$ & $3 \mid 9.1$ \\
\hline PIERIDAE & 200 & $67 \mid 34$ & 517.5 \\
\hline Pseudopontiinae & 5 & $2 \mid 40$ & \\
\hline Coliadinae & 14 & $8 \mid 57$ & \\
\hline Pierinae & 181 & $57 \mid 32$ & \\
\hline LYCAENIDAE & 1837 & $210 \mid 11$ & $18 \mid 8.6$ \\
\hline Miletinae & 119 & $11 \mid 9$ & \\
\hline Poritiinae & 658 & 5318 & \\
\hline Theclinae & 301 & $42 \mid 14$ & \\
\hline Aphnaeinae & 260 & 2118 & \\
\hline Polyommatinae & 496 & $83 \mid 17$ & \\
\hline RIODINIDAE & 15 & $4 \mid 27$ & $0 \mid 0$ \\
\hline NYMPHALIDAE & 1634 & $344 \mid 21$ & 2617.6 \\
\hline Libytheinae & 5 & $2 \mid 40$ & \\
\hline Danainae & 26 & $9 \mid 35$ & \\
\hline Satyrinae & 347 & $50 \mid 14$ & \\
\hline Charaxinae & 190 & $56 \mid 30$ & \\
\hline Apaturinae & 3 & $1 \mid 33$ & \\
\hline Nymphalinae & 73 & $35 \mid 48$ & \\
\hline Cyrestinae & 1 & $1 \mid 100$ & \\
\hline Biblidinae & 31 & $16 \mid 52$ & \\
\hline Limenitidinae & 702 & $97 \mid 14$ & \\
\hline Heliconiinae & 256 & $77 \mid 30$ & \\
\hline TOTALS & 4405 & $792 \mid 18$ & \\
\hline
\end{tabular}

terflies and skippers have been collected in Angola have been determined (Mendes et al. 2013b), some localities previously reported for a few species remain untraced despite searches by us, using the detailed maps of the Junta de Investigações do Ultramar (JIU 1948-1963).

The accumulation of knowledge in regard to Angolan butterflies has been constrained by several factors. The two largest Angolan entomological collections, deposited in the Museu do Dundo (Lunda-Norte) and in the Instituto de Investigação Agronómica (Huambo), have never been studied in detail. In addition little fieldwork was carried out in Angola during the post-independence period because of the protracted civil war. Finally, the vastness of the country and the difficulty in accessing many remote regions has impeded progress. 


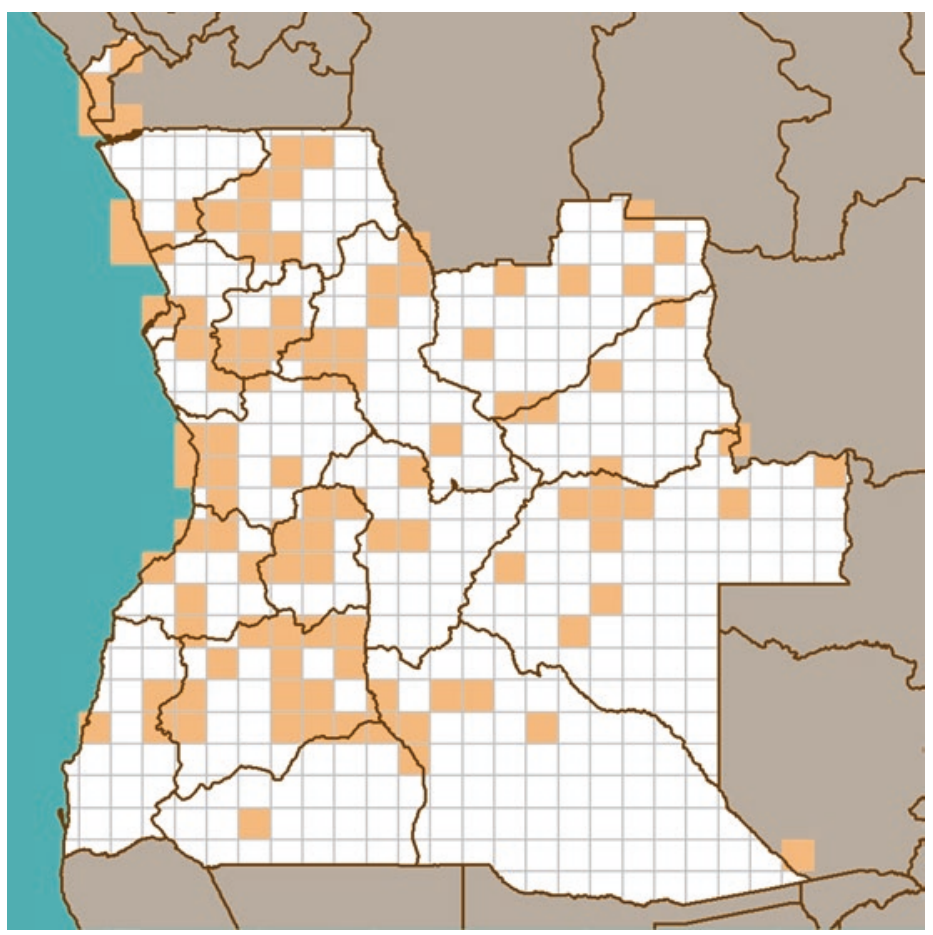

Fig. 10.2 Map of Angola showing, marked in orange, the known areas surveyed for the Papilionoidea from the beginning of their study, in the nineteenth century to the present day - each square ca. $33 \times 33 \mathrm{~km}$. The collecting pressure varies across the country, from 'squares' where samples were obtained only once, in passing, to others where the collectors were based for months

Until recently the Hesperiidae (skippers) were placed in the superfamily Hesperioidea, separate from the rest of the butterflies, which were placed in the superfamily Papilionoidea. However, today the skippers and butterflies are all placed in the Papilionoidea (e.g. Heikkilä et al. 2012). The classification used for butterflies in this chapter is based on Williams (2018), Espeland et al. (2018) and Dhungel and Wahlberg (2018). Six families of butterflies are represented in Angola, namely Papilionidae, Hesperiidae, Pieridae, Riodinidae, Lycaenidae and Nymphalidae.

\section{History of Research on the Papilionoidea of Angola}

The first known reference to butterflies obtained in Angola is by Latreille and Godart (1819), who reported the presence of Colotis euippe (Linnaeus, 1758) and described Acraea parrhasia servona. In the decade between 1871 and 1880, Druce (1875) reported about 90 species from Angola for the first time, a number of these being descriptions of species new to science. By the end of the nineteenth century a total of 214 butterfly taxa were known from Angola. 
The first contributions to our knowledge of Angolan butterflies by Portuguese researchers were only made in the middle nineteen hundreds. These were the result of the activities in Angola of the Centro de Zoologia (CZ) of the Junta de Investigações do Ultramar, coordinated by its first director Fernando Frade. In 2014 this research institution was renamed the Instituto de Investigação Científica Tropical (IICT). Working from these large zoological collections, as well as from further specimens obtained in Angola by Amélia Bacelar (1948, 1956, 1958a, b, 1961) and Miguel Ladeiro (1956), considerably expanded the list of Angolan butterflies. Most of this material, obtained during colonial times, was stored in Lisbon, with corrections to the published identifications only being made recently. All of this material has now been integrated into the collections of the Museu Nacional de História Natural e da Ciência (MUHNAC). Albert Monard (1956) of the La Chauxde-Fonds Swiss Museum also studied other material obtained by the CZ missions. Significant contributions in the twentieth century were also made by Weymer (1901) on the southern Angolan species, and by Evans (1937) on the Hesperiidae. All of the then known Angolan butterflies were listed by Aurivillius (in Seitz) in 1928. All of the Angolan Charaxinae were dealt with by Henning in his 1988 book on the African taxa of this family. The 339 taxa added to the faunal list during the twentieth century brought the total to 553 known butterfly taxa for Angola.

During the first 18 years of the twenty-first century, 239 further taxa were added to the total. In the first decade of the present century, most of the new information was due to several contributions by Libert $(1999,2000,2004)$ on the Lycaenidae, and by Gardiner (2004). The latter author listed taxa from the southeastern Cuando Cubango province, which borders the Caprivi Strip of Namibia. Cuando Cubango and the easternmost province of Moxico are the only provinces in Angola with Zambezian fauna. To these taxa we add our own contributions (Bivar-de-Sousa and Mendes 2006, 2007, 2009a, b; Mendes and Bivar-de-Sousa 2006a, b, 2007a, b, 2009a, b, c, d). Over the last 8 years 33 species were described as new or recorded for the first time from Angola by Mendes and Bivar-de-Sousa (2012, 2017) Mendes et al. (2013a, 2017, 2018), Bivar-de-Sousa and Mendes (2014) and Bivar-de-Sousa et al. (2017), Turlin and Vingerhoedt (2013) and Pierre and Bernaud (2013). Finally, 66 further taxa are now recorded as faunistic novelties for the country (Appendix). The current total number of butterfly taxa for Angola now stands at 792.

\section{Sources Consulted for the Checklist}

In preparing this revised checklist of the Papilionoidea, the following collections of Angolan butterflies held by institutions in Portugal were examined: Museu Nacional de História Natural e da Ciência (MUHNAC) in Lisbon, Museu de História Natural da Universidade do Porto (MHNC-UP), Liceu Nun'Álvares in the Caldas da Saúde and the Singeverga Order of St Benedict Abbey in Areias. Major contributions to these collections were made by A Bivar-de-Sousa (Luanda district and CuanzaNorte, Cuanza-Sul and Moxico Provinces), António Figueira, (northwestern Angola), Mário Macedo (northern Angola), Passos de Carvalho (Huambo and 
Cuanza-Norte Provinces), Carneiro Mendes and Pessoa Guerreiro. The Angolan insect collection of Nozolino de Azevedo (mainly Huambo Province), maintained and made available by his widow, was also studied.

The collections in the MUHNAC in Lisbon were destroyed by a fire in March 1978. However, prior to the fire BS had studied some of the material and published his findings. In 1995 LM studied the collections, mainly of Barros Machado and Luna de Carvalho, in the Dundo Museum in Angola but there was insufficient time to do a detailed analysis. We did not inspect the entomological collections in the former Instituto de Investigação Agronómica de Angola, collected mainly by Passos de Carvalho, but they are apparently in good condition. No entomological collections were found by LM, in 1995 and 2013, at the Museu de História Natural de Luanda. Material collected from 2010 to 2014 by Ruben Capela and Carmen VanDúnen Santos of Agostinho Neto University, Luanda and Artur Serrano of the Faculty of Sciences, Lisbon University, was examined by us.

In addition, images of live specimens published by Lautenschläger and Neinhhuis (2014) were examined, as were several images presented by Jorge Palmeirim of Lisbon University and Pedro Vaz Pinto of the Kissama Foundation.

\section{Taxa Excluded from the Checklist}

A number of taxa have erroneously been reported to occur in Angola. This was due mainly to probable misidentifications or mislabelled specimens. Some older records are omitted because the known range of the taxon is unlikely to include Angola. A list of the omitted taxa is given below.

- Hesperiidae: Eretis djaelaelae (Wallengren, 1857), Metisella metis (Linnaeus, 1764), Kedestes chaca (Trimen, 1873), Platylesches chamaeleon (Mabille, 1891).

- Papilionidae: Papilio menestheus Drury, 1773, Graphium taboranus (Oberthür, 1886), Graphium (Arisbe) junodi (Trimen, 1893).

- Pieridae: Eurema brigitta (Stoll, 1780), Colotis chrysonome (Klug, 1829), Colotis ephyia (Klug, 1829), Belenois theora (Doubleday, 1846), Mylothris rubricosta (Mabille, 1890), Mylothris similis Lathy, 1906.

- Lycaenidae: Telipna acraea (Westwood, 1851), Cooksonia abri Collins \& Larsen, 2008, Mimacraea darwinia Butler, 1872, Liptena bassae Bethune-Baker, 1926, Aethiopana honorius honorius (Fabricius, 1793), Stempfferia uniformis (Kirby, 1887), Stempfferia dorothea (Bethune-Baker, 1904), Oxylides faunus (Drury, 1773), Dapidodigma hymen (Fabricius, 1775), Aloeides molomo (Trimen, 1870), Leptomyrina lara (Linnaeus, 1764), Deudorix livia (Klug, 1834), Neurellipes onias (Hulstaert, 1924), Zintha hintza (Trimen, 1864).

- Riodinidae: Afriodinia caeca semicaeca (Riley, 1932), Afriodinia gerontes (Fabricius, 1781).

- Nymphalidae: Bicyclus milyas (Hewitson, 1864), Ypthima congoana Overlaet, 1955, Charaxes jahlusa argynnides Westwood, 1864, Junonia touhilimasa 
Vuillot, 1892, Neptis continuata Holland, 1892, Neptis strigata Aurivillius, 1894, Evena oberthueri (Karsch, 1894), Euriphene atrovirens (Mabille, 1878), Bebearia mardania (Fabricius, 1793), Euphaedra morini Hecq, 1983, Euphaedra xypete (Hewitson, 1865), Euphaedra campaspe (Felder \& Felder, 1867), Euphaedra inanum (Butler, 1873), Euphaedra eupalus (Fabricius, 1781).

\section{A Revised Checklist of the Papilionoidea of Angola}

A revised and annotated checklist of the Papilionoidea of Angola (Appendix) confirms the presence of at least 792 taxa in the country. Their presence is based mainly on verification by the authors of this chapter. Some taxa, recorded by other authors, are accepted because they were, with rare exceptions, reported by more than one author, are based on reliable literature records, or because Angola falls within their putative geographical range. In the Checklist, the first reference to their occurrence in Angola is given, followed by the sources of validation of the record and their preferred habitat(s). Occasionally more than one subspecies of a particular species occurs in the country. This is due to both the size and ecological diversity of Angola. A number of forests, especially gallery-forests, are independent of each other as are the fragmented forests of the Angolan Escarpment. In addition, the southeastern parts of Moxico and the Cuando Cubango provinces are part of the Zambesi Basin; consequently their fauna has affinities with that of eastern Africa.

As far as habitats are concerned the great majority of the Angolan Papilionoidea, as might be expected, occur in forest, both wet and dry (Appendix). However, the Hesperiidae and Pieridae appear to be almost as diverse in moist woodland (miombo) and dry woodland as they are in forest. The number of Pieridae in dry woodland and miombo is similar, while the number of species in dry woodland, arid shrubland and grassland surpasses that of wet forest. The subfamily Nymphalinae is more diverse in miombo than forest and equally diverse in savanna. The Heliconiinae (Nymphalidae) in savanna are almost as diverse as they are in wet forest.

\section{Composition, Diversity and Endemism}

All six families and all of the subfamilies (except the Lycaeninae, Leach, 1815) of Afrotropical butterflies are represented in Angola (Table 10.1).

One genus and 56 species/subspecies of Papilionoidea are endemic to Angola, many of which were described over the last few decades. The endemic genus Mashunoides, Mendes and Bivar-de-Sousa 2009a, b, c, d (Nymphalidae: Satyrinae) is confined to Cuando Cubango Province, in the ecotone between miombo and savanna/dry woodland mosaic. Endemism rates for Angolan butterfly families are highest for the Papilionidae and Lycaenidae and lowest for the Hesperiidae and Riodinidae (Tables 10.1 and 10.2). Examples of endemic species are illustrated in Fig. 10.3. 
Table 10.2 Endemic butterfly species and subspecies in Angola

\begin{tabular}{|c|c|c|}
\hline Family & Endemic species & Endemic subspecies \\
\hline Hesperiidae & $\begin{array}{l}\text { Eagris multiplagata } \\
\text { Abantis bergeri }\end{array}$ & $\begin{array}{l}\text { Calleagris jamesoni ansorgei } \\
\text { Eretis herewardi rotundimacula } \\
\text { Spialia colotes colotes }\end{array}$ \\
\hline Papilionidae & $\begin{array}{l}\text { Papilio bacelarae } \\
\text { Papilio chitondensis }\end{array}$ & Papilio macinnoni benguellae \\
\hline Pieridae & Mylothris carvalhoi & $\begin{array}{l}\text { Appias epaphia angolensis } \\
\text { Appias phaola uigensis } \\
\text { Appias sylvia ribeiroi } \\
\text { Mylothris spica gabela }\end{array}$ \\
\hline Lycaenidae & $\begin{array}{l}\text { Alaena rosei } \\
\text { Cooksonia nozolinoi } \\
\text { Falcuna lacteata } \\
\text { Deloneura barca } \\
\text { Aloeides angolensis } \\
\text { Zeritis krystyna } \\
\text { Cupidesthes vidua } \\
\text { Uranothauma nozolinoi Lepidochrysops } \\
\text { ansorgei } \\
\text { Lepidochrysops flavisquamosa } \\
\text { Lepidochrysops fulvescens } \\
\text { Lepidochrysops hawker } \\
\text { Lepidochrysops nacrescens } \\
\text { Lepidochrysops reichenowi }\end{array}$ & $\begin{array}{l}\text { Liptena homeyeri straminea } \\
\text { Falcuna libyssa angolensis } \\
\text { Cigarits modestus modestus } \\
\text { Leptomyrina henningi } \\
\text { angolensis }\end{array}$ \\
\hline Nymphalidae & $\begin{array}{l}\text { Brakefieldia angolensis } \\
\text { Brakefieldia ochracea } \\
\text { Neita bikuarica } \\
\text { Mashunoides carneiromendesi } \\
\text { Charaxes figuerai } \\
\text { Charaxes ehmckei } \\
\text { Precis larseni } \\
\text { Bebearia hassoni } \\
\text { Euphaedra divoides } \\
\text { Euphaedra uigensis } \\
\text { Acraea bellona } \\
\text { Acraea lapidorum, } \\
\text { Acraea onerata }\end{array}$ & $\begin{array}{l}\text { Amauris crawshayi angola, } \\
\text { Amauris dannfelti dannfelti } \\
\text { Charaxes fulvescens } \\
\text { rubenarturi, } \\
\text { Charaxes macclouni carvalhoi, } \\
\text { Charaxes lucretius saldanhai } \\
\text { Charaxes jahlusa angolensis } \\
\text { Charaxes minor karinae } \\
\text { Charaxes trajanus bambi } \\
\text { Palla ussheri hassoni } \\
\text { Sevenia occidentalium penricei } \\
\text { Euphaedra harpalyce } \\
\text { commineura } \\
\text { Acraea violarum anchietai }\end{array}$ \\
\hline
\end{tabular}

\section{Conservation}

Because butterflies are sensitive to changing environmental conditions and are taxonomically well known, they are valuable as indicators of ecological dynamics. They are also key drivers of ecological processes. In particular, adult butterflies are active pollinators of many plants and the imagos and larvae are an important source of nutrition for a diverse range of vertebrate and invertebrate predators and insect parasitoids. Their conservation importance is also due to their positive and occasionally negative economic impacts. Although humans utilise mainly moth caterpillars as a 

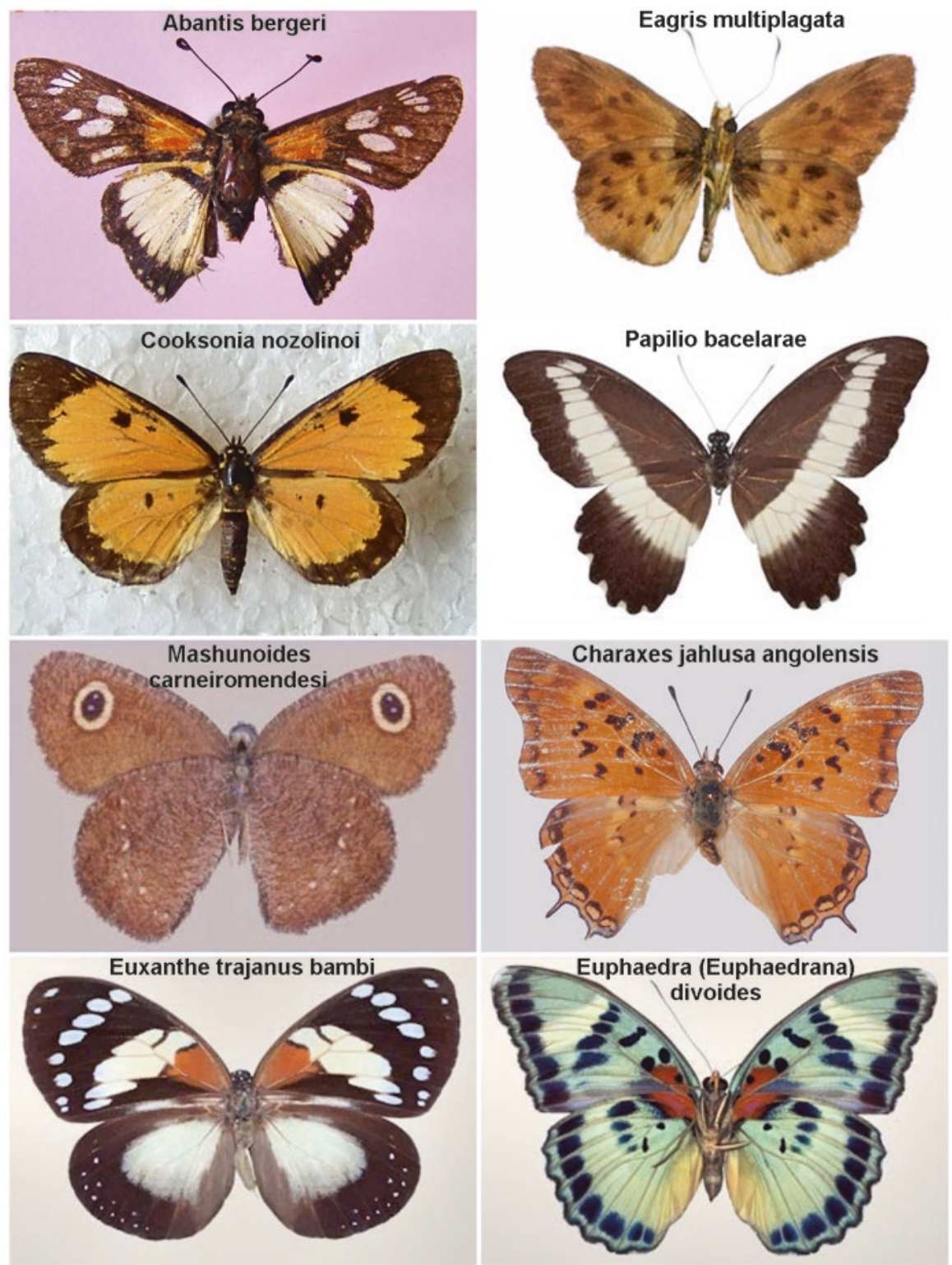

Fig. 10.3 The holotype specimens of endemic Angolan papilionoidea: Left to right, top to bottom ( $V$ Ventral, D Dorsal): 1. Abantis bergeri male D (Mendes and Bivar-de-Sousa 2009a, b, c, d), 2. Eagris multiplagata male V (Bivar-de-Sousa and Mendes 2007), 3. Cooksonia nozolinoi female D (Mendes and Bivar-de-Sousa 2007), 4. Papilio bacelarae male D (Bivar-de-Sousa and Mendes 2009a, b), 5. Mashunoides carneiromendesi male V (Mendes and Bivar-de-Sousa 2009a, b, c, d), 6. Charaxes jahlusa angolensis male D (Mendes et al. 2017), 7. Euxanthe trajanus bambi male D (Bivar-de-Sousa and Mendes 2006), 8. Euphaedra (Euphaedrana) divoides male V (Bivar- deSousa and Mendes 2018) 
food source, the larvae of the skipper Coeliades libeon is much appreciated. A limited number of butterfly species are agricultural pests, including Papilio demodocus (young citrus orchards), Lampides boeticus (cultivated Leguminosae) and Acraea acerata (sweet-potatoes). A few species, such as Pyrrhochalcia iphis and Zophopetes dysmephila, may cause damage in coconut and oil-palm plantations.

In terms of species of conservation concern, information on the status of Angolan butterflies is very limited. Many species of Angolan butterflies are obviously abundant and widespread, both within and outside the country. Those taxa that appear to be rare and/or more localised may be genuinely rare or local but this may simply reflect a paucity of information. This makes it difficult or impossible to propose rational conservation measures at present. The urgent need for more fieldwork, particularly in regard to the endemic taxa, is thus highlighted. In the meantime habitat conservation, especially with respect to isolated forest patches, can be considered as part of a wider effort to conserve both the fauna and flora of the country.

\section{Potential Future Discoveries and Research}

Considering the number of taxa new to science described in the last few decades there are almost certainly further undiscovered butterfly taxa in Angola. Vast areas of the country remain unexplored, mainly because of inaccessibility and postindependence political instability. Not only will new taxa be found but also known taxa from bordering countries will be added to the list of Angolan butterflies during future fieldwork. This work will also improve our knowledge in regard to the distribution of the taxa in the country. Finally, almost nothing is known about the habitats, behaviours, early stages and larval host plants of Angolan butterflies, making these fertile areas for future research on the fauna. More information concerning all the endemic taxa is urgently needed in order to determine conservation priorities.

\section{Appendix}

Checklist of Papilionoidea recorded from Angola (by Family I Sub-family).

For the species' authors and references, Bivar-de-Sousa is abbreviated as BS and Mendes as M; $(*)=$ Taxa with Angola as type locality; $\mathbf{0}=$ Taxa now reported as new to Angola; $\boldsymbol{\Delta}=$ Previous references existing but not traced (several species when reported from Angola were assigned under names today considered synonyms, others at species level - the corresponding Angolan endemics were described later).

$\mathrm{V}=$ Validation of the taxon - 1: Endemic (restricted to Angola); 2: Restricted to Angola and to the neighbouring northern countries - Gabon, Congo and/or DRC; 3: Restricted to Angola and to the neighbouring eastern countries - southern DRC (former Shaba) and/or Zambia; 4: Restricted to Angola and to the southern and 
south-eastern neighbouring countries - Namibia and/or Botswana; 5: Species/subspecies with material studied by the authors; 6: Taxa exclusively known in the country from previously collected unstudied material - known from bibliographic references only. H - Preferred habitat: A: Humid forest - primary and secondary wet forest, gallery and riverine forest, forest edge; B: Dry forest, including dry forest and savanna mosaics; C: Brachystegia woodland (miombo) and other woodland; D: Mixed savanna with or without trees; E: Swampy areas, including the northeastern moist thicket and savanna mosaic; F: Arid shrubland and grassland; G: Rocky hillsides; H: Ubiquitous or almost ubiquitous; X: Caterpillars (almost) monophagous, the imagos range dependent on the presence of host-plants

\begin{tabular}{|c|c|c|c|}
\hline Taxon & First reference for Angola & $\mathrm{V}$ & $\mathrm{H}$ \\
\hline \multicolumn{4}{|l|}{ HESPERIIDAE | Coeliadinae } \\
\hline Coeliades bixana (Evans, 1940) & Evans, 1937, as C. bixae & 6 & A \\
\hline Coeliades c. chalybe (Westwood, 1852) & Evans, 1937 & 5 & A \\
\hline Coeliades libeon (Druce, 1875) & Druce, $1875(*)$ & 5 & $\mathrm{~B}, \mathrm{C}$ \\
\hline Coeliades f. forestan (Stoll, 1782) & Ladeiro, 1959 & 5 & $\mathrm{H}$ \\
\hline Coeliades hanno (Plötz, 1879) & Evans, 1937 & 6 & A \\
\hline Coeliades pisistratus (Fabricius, 1793) & Bacelar, 1948 & 6 & $\mathrm{D}$ \\
\hline $\begin{array}{l}\text { Pyrrhochalcia iphis dejongi (Collins \& } \\
\text { Larsen, 2008) }\end{array}$ & Bacelar, 1956, as P. iphis & 2,5 & A \\
\hline \multicolumn{4}{|l|}{ HesperiIdae | Pyrginae } \\
\hline Apallaga rutilans (Mabille, 1877) & M et al., 2013a & 5 & A \\
\hline Apallaga h. homeyeri (Plötz, 1880) & Plötz, $1880\left(^{*}\right)$ & 6 & A \\
\hline $\begin{array}{l}\text { Celaenorrhinus p. proxima (Mabille, } \\
\text { 1877) }\end{array}$ & M \& BS, 2009a, b, c, d & 5 & A \\
\hline Tagiades flesus (Fabricius, 1781) & Evans, 1937 & 5 & A,B \\
\hline Eagris lucetia (Hewitson, 1875) & Aurivillius, 1928 & 5 & A \\
\hline $\begin{array}{l}\text { Eagris decastigma fuscosa (Holland, } \\
1893\end{array}$ & M et al., 2013a & 5 & A \\
\hline $\begin{array}{l}\text { Eagris tigris liberti (Collins \& Larsen, } \\
\text { 2005) }\end{array}$ & Evans, 1937, as E. tigris & 6 & A \\
\hline Eagris h. hereus (Druce, 1875) & Druce, $1875(*)$ & 6 & A \\
\hline Eagris t. tetrastigma (Mabille, 1891) & M et al., 2013a & 5 & A \\
\hline Eagris multiplagata (BS \& M, 2007) & BS \& M, $2007(*)$ & 1,5 & A \\
\hline $\begin{array}{l}\text { Ortholexis hollandi (Druce, 1909) f. } \\
\text { karschi (Evans, 1937) }\end{array}$ & Evans, 1937 & 6 & A \\
\hline Calleagris hollandi (Butler, 1897) & Evans, 1937 & 5 & $\mathrm{C}$ \\
\hline $\begin{array}{l}\text { Calleagris jamesoni ansorgei (Evans, } \\
\text { 1951) }\end{array}$ & 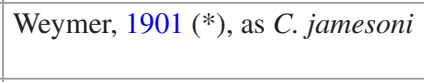 & 1,5 & $\mathrm{C}$ \\
\hline Calleagris l. lacteus (Mabille, 1877) & Bacelar, 1961 & 6 & A \\
\hline Eretis lugens (Rogenhofer, 1891) & Larsen, 2005 & 5 & $\mathrm{D}$ \\
\hline $\begin{array}{l}\text { Eretis herewardi rotundimacula (Evans, } \\
\text { 1937) }\end{array}$ & Evans, 1937 (*) $^{*}$ & 1,5 & $\mathrm{C}$ \\
\hline Eretis melania (Mabille, 1891) & Evans, 1937 & 5 & C,D \\
\hline
\end{tabular}




\begin{tabular}{|c|c|c|c|}
\hline Taxon & First reference for Angola & $\mathrm{V}$ & $\mathrm{H}$ \\
\hline Sarangesa loelius (Mabille, 1877) & Bacelar, 1948 & 5 & $\mathrm{C}$ \\
\hline Sarangesa l. lucidella (Mabille, 1891) & M et al., 2013a & 5 & $\mathrm{D}$ \\
\hline Sarangesa motozi (Wallengren, 1857) & Aurivillius, 1928 & 5 & $\mathrm{~A}, \mathrm{C}$ \\
\hline Sarangesa phidyle (Walker, 1870) & Evans, 1937 & 5 & $\mathrm{~B}, \mathrm{D}$ \\
\hline Sarangesa s. seineri (Strand, 1909) & Evans, 1937 & 5 & $\mathrm{D}$ \\
\hline $\begin{array}{l}\text { Sarangesa p. pandaensis (Joicey \& } \\
\text { Talbot, 1921) }\end{array}$ & Evans, 1937 & 3,5 & $\mathrm{C}$ \\
\hline Sarangesa bouvieri (Mabille, 1877) & Evans, 1937 & 6 & B \\
\hline Sarangesa brigida sanaga (Miller, 1964) & M et al., 2013a & 5 & A \\
\hline Sarangesa maculata (Mabille, 1891) & M et al., 2013a & 5 & $\mathrm{~A}, \mathrm{~B}, \mathrm{C}$ \\
\hline Triskelionia tricerata (Mabille, 1891 ) & M et al., 2013a & 6 & A \\
\hline $\begin{array}{l}\text { Caprona cassualala (Bethune-Baker, } \\
\text { 1911) }\end{array}$ & Bethune-Baker, $1911(*)$ & 5 & $\mathrm{D}, \mathrm{F}$ \\
\hline Caprona pillaana (Wallengren, 1857) & M \& BS, 2013a & 5 & $\mathrm{D}, \mathrm{F}$ \\
\hline Netrobalane canopus (Trimen, 1864) & M \& BS, 2013a & 5 & $\mathrm{D}, \mathrm{A}$ \\
\hline Leucochitonea levubu (Wallengren, 1857) & Weymer, 1901 & 5 & $\mathrm{C}, \mathrm{D}$ \\
\hline Abantis tettensis (Hopffer, 1855) & Aurivillius, 1928 & 5 & $\mathrm{C}, \mathrm{D}$ \\
\hline Abantis bergeri (M \& BS, 2009a, b, c, d) & M \& BS, 2009a, b, c, d (*) & 1,5 & $\mathrm{C}$ \\
\hline Abantis paradisea (Butler, 1870$)$ & Weymer, 1901 & 6 & $\mathrm{C}$ \\
\hline Abantis zambesiaca (Westwood, 1874) & Weymer, 1901 & 5 & $\mathrm{D}, \mathrm{C}$ \\
\hline Abantis contigua (Evans, 1937) & Evans, 1937 & 5 & $\mathrm{C}$ \\
\hline Abantis venosa (Trimen, 1889$)$ & M et al., 2013a & 5 & $\mathrm{C}$ \\
\hline Abantis vidua (Weymer, 1901) & Weymer, 1901 & 3 & $\mathrm{C}$ \\
\hline Spialia m. mafa (Trimen, 1870) & Weymer, 1901 & 6 & $\mathrm{D}$ \\
\hline Spialia spio (Linnaeus, 1764) & Weymer, 1901 & 5 & $\mathrm{D}$ \\
\hline Spialia delagoae (Trimen, 1898) & Larsen, 1996 & 5 & $\mathrm{D}$ \\
\hline Spialia c. colotes $($ Druce, 1875$)$ & Druce, $1875(*)$ & 1,5 & $\mathrm{D}, \mathrm{B}$ \\
\hline $\begin{array}{l}\text { Spialia colotes transvaaliae (Trimen, } \\
\text { 1889) }\end{array}$ & $\mathbf{\Delta}$ & 5 & $\mathrm{D}$ \\
\hline Spialia ferax (Wallengren, 1863) & M et al., 2013a & 5 & C,D \\
\hline Spialia dromus (Plötz, 1884) & Weymer, 1901 & 5 & $\mathrm{C}, \mathrm{D}$ \\
\hline Spialia p. ploetzi (Aurivillius, 1891) & Evans, 1937 , as $S$. rebeli & 6 & A \\
\hline Spialia secessus (Trimen, 1891) & Trimen, $1891(*)$ & 5 & $\mathrm{~F}$ \\
\hline Gomalia e. elma (Trimen, 1862) & Aurivilius, 1928 & 5 & $\mathrm{D}$ \\
\hline \multicolumn{4}{|l|}{ HESPERIIDAE | Heteropterinae } \\
\hline Metisella m. midas (Butler, 1894) & Monard, 1956 & 5 & $\mathrm{E}$ \\
\hline Metisella a. angolana (Karsch, 1896) & Karsch, $1896\left(^{*}\right)$ & 5 & B \\
\hline Metisella willemi (Wallengren, 1857) & M et al., 2013a, b & 5 & $\mathrm{C}$ \\
\hline Metisella meninx (Trimen, 1873) & Evans, 1937 & 6 & $\mathrm{E}$ \\
\hline Lepella lepeletier (Latreille, 1824) & Druce, 1875 & 5 & $\mathrm{~F}$ \\
\hline \multicolumn{4}{|l|}{ HESPERIIDAE | Hesperiinae } \\
\hline Astictopterus abjecta (Snellen, 1872) & Snellen, $1872(*)$ & 6 & A \\
\hline Astictopterus punctulata (Butler, 1895) & M et al., 2013a & 5 & $\mathrm{C}$ \\
\hline Kedestes mohozutza (Wallengren, 1857) & Monard, 1956 & 5 & $\mathrm{D}$ \\
\hline
\end{tabular}




\begin{tabular}{|c|c|c|c|}
\hline Taxon & First reference for Angola & V & $\mathrm{H}$ \\
\hline Kedestes nerva paola (Plötz, 1884) & Plötz, $1884(*)$ & 5 & A \\
\hline Kedestes brunneostriga (Plötz, 1884) & Plötz, $1884(*)$ & 5 & $\mathrm{C}$ \\
\hline Kedestes straeleni (Evans, 1956) & M et al., 2013a & 5 & $\mathrm{C}$ \\
\hline Kedestes l. lema $($ Neave, 1910$)$ & Evans, 1937 & 3 & $\mathrm{C}$ \\
\hline Kedestes callicles (Hewitson, 1868) & Aurivillius, 1928 & 5 & $\mathrm{C}$ \\
\hline Gorgyra mocquerysii (Holland, 1896) & Evans, 1937 & 5 & A \\
\hline Gorgyra diversata (Evans, 1937) & Evans, 1937 & 6 & A \\
\hline $\begin{array}{l}\text { Ceratrichia nothus makomensis (Strand, } \\
\text { 1913) }\end{array}$ & M et al., 2013a & 5 & A \\
\hline Ceratrichia punctata (Holland, 1896) & Evans, 1937 & 6 & A \\
\hline Teniorhinus harona (Westwood, 1881) & Weymer, 1901, as Oxypalpus ruso & 5 & $\mathrm{C}$ \\
\hline Teniorhinus ignita (Mabille, 1877) & Monard, 1956 & 5 & B \\
\hline Pardaleodes edipus (Stoll, 1781) & Bacelar, 1948 & 6 & A \\
\hline Pardaleodes $i$. incerta (Snellen, 1872) & Evans, 1937 & 5 & A,D \\
\hline $\begin{array}{l}\text { Pardaleodes sator pusiella (Mabille, } \\
\text { 1877) }\end{array}$ & Mabille, $1877(*)$ & 5 & A \\
\hline Pardaleodes t. tibullus (Fabricius, 1793) & M \& BS, 2009 & 5 & A \\
\hline Acada biseriata (Mabille, 1893) & Evans, 1937 & 5 & $\mathrm{C}$ \\
\hline Parosmodes lentiginosa (Holland, 1896) & Evans, 1937 & 5 & A \\
\hline Parosmodes m. morantii (Trimen, 1873) & Weymer, 1901 & 5 & $\mathrm{C} / \mathrm{D}$ \\
\hline Osmodes laronia (Hewitson, 1868) & Druce, 1875 & 6 & A \\
\hline Osmodes thora (Plötz, 1884) & Evans, 1937 & 6 & A \\
\hline Acleros mackenii olaus (Plötz, 1884) & Druce, 1875 , the species & 5 & A \\
\hline Acleros nigrapex (Strand, 1913) & M et al., 2013a & 5 & A \\
\hline Acleros ploetzi (Mabille, 1889) & M \& BS, 2009 & 5 & A \\
\hline Semalea arela (Mabille, 1891) & M et al., 2013a & 5 & A \\
\hline Semalea pulvina (Plötz, 1879) & M \& BS, 2009 & 5 & A \\
\hline Semalea sextilis (Plötz, 1886) & M et al., 2013a & 5 & A \\
\hline Hypoleucis o. ophiusa (Hewitson, 1866) & M \& BS, 2009 & 5 & A \\
\hline Meza indusiata (Mabille, 1891) & Larsen, 2005 & 5 & A,B \\
\hline Meza meza (Hewitson, 1877) & Hewitson, $1877(*)$ & 5 & A \\
\hline Meza c. cybeutes (Holland, 1894) & Evans, 1937 & 6 & A \\
\hline Meza mabillei (Holland, 1893) & M et al., 2013a & 5 & A \\
\hline Paronymus ligora (Hewitson, 1876) & Hewitson, $1876(*)$ & 6 & A \\
\hline Andronymus n. neander (Plötz, 1884) & Evans, 1937 & 5 & A \\
\hline Andronymus c. caesar (Fabricius, 1793) & Aurivillius, 1928 as A. caesar & 5 & A \\
\hline $\begin{array}{l}\text { Andronymus caesar philander (Hopffer, } \\
1855 \text { ) }\end{array}$ & Aurivillius, 1928 as A. caesar & 6 & A \\
\hline Andronymus hero (Evans, 1937) & Evans, 1937 & 5 & A \\
\hline Andronymus helles (Evans, 1937) & Evans, 1937 & 5 & A \\
\hline Chondrolepis niveicornis (Plötz, 1882) & Plötz, $1882(*)$ & 5 & $\mathrm{E}$ \\
\hline Zophopetes dysmephila (Trimen, 1868) & Aurivillius, 1928, as Z. schultzi & 6 & A,D \\
\hline Zophopetes cerymica (Hewitson, 1867) & M \& BS, 2009 & 5 & $\mathrm{X}$ \\
\hline Gamia shelleyi (Sharpe, 1890) & M et al., 2013a & 5 & A \\
\hline
\end{tabular}




\begin{tabular}{|c|c|c|c|}
\hline Taxon & First reference for Angola & $\mathrm{V}$ & $\mathrm{H}$ \\
\hline Gretna cylinda (Hewitson, 1876) & Aurivillius, $1928(*)$ & 5 & $\mathrm{~A}, \mathrm{C}$ \\
\hline Gretna waga (Plötz, 1886) & M et al., 2013a & 5 & $\mathrm{~A}, \mathrm{C}$ \\
\hline Pteroteinon laufella (Hewitson, 1868) & Druce, 1875 & 5 & $\mathrm{~B}, \mathrm{C}$ \\
\hline Pteroteinon caenira (Hewitson, 1867) & M \& BS, 2009 & 5 & B \\
\hline $\begin{array}{l}\text { Pteroteinon concaenira (Belcastro \& } \\
\text { Larsen, 1996) }\end{array}$ & M et al., 2013a & 6 & B \\
\hline Leona maracanda (Hewitson, 1876) & Hewitson, $1876(*)$ & 5 & A \\
\hline Caenides dacela (Hewitson, 1876) & Williams, 2007 & 5 & $\mathrm{~A}$ \\
\hline Monza cretacea (Snellen, 1872) & Evans, 1937 & 5 & $\mathrm{~B}$ \\
\hline Fresna nyassae (Hewitson, 1878) & Aurivillius, 1928 & 5 & $\mathrm{C}, \mathrm{D}$ \\
\hline Platylesches langa (Evans, 1937) & M et al., 2013a & 6 & $\mathrm{C}$ \\
\hline Platylesches moritili (Wallengren, 1857) & Trimen, 1891 & 5 & C,D \\
\hline Platylesches robustus (Neave, 1910) & M et al., 2013a & 6 & $\mathrm{C}$ \\
\hline Platylesches cf. batangae (Holland, 1894) & $\Delta$ & 5 & $\mathrm{~B}$ \\
\hline Brusa allardi (Berger, 1967) & M et al., 2013a & 6 & $\mathrm{C}$ \\
\hline Zenonia zeno (Trimen, 1864) & Plötz, 1883, as Hesperia coanza & 5 & $\mathrm{~A}, \mathrm{C}$ \\
\hline Pelopidas m. mathias (Fabricius, 1798) & Evans, 1937 & 5 & $\mathrm{C}$ \\
\hline Pelopidas thrax (Hübner, 1821) & Gardiner, 2004 & 5 & $\mathrm{D}$ \\
\hline Borbo fallax (Gaede, 1916) & M \& BS, 2009a, b, c, d & 5 & $\mathrm{D}$ \\
\hline Borbo fanta (Evans, 1937) & Evans, 1937 & 5 & $\mathrm{D}$ \\
\hline Borbo sirena $($ Evans, 1937$)$ & M et al., 2013a & 5 & $\mathrm{~A}, \mathrm{~B}, \mathrm{C}$ \\
\hline Borbo b. borbonica (Boisduval, 1833) & $\Delta$ & 5 & $\mathrm{D}$ \\
\hline Borbo detecta (Trimen, 1893) & Weymer, 1901 & 5 & $\mathrm{~B}$ \\
\hline Larsenia gemella (Mabille, 1884) & Evans, 1937 & 5 & $\mathrm{D}$ \\
\hline Borbo micans (Holland, 1896) & M \& BS, 2009 & 5 & $\mathrm{E}$ \\
\hline Larsenia perobscura (Druce, 1812) & M et al., 2013a & 5 & $\mathrm{D}$ \\
\hline Borbof. fatuellus (Hopffer, 1855) & M \& BS, 2009 & 5 & $\mathrm{~B}, \mathrm{C}$ \\
\hline Larsenia holtzi (Plötz, 1883) & Plötz, $1883(*)$ & 5 & $\mathrm{D}$ \\
\hline Parnara monasi (Trimen, 1889) & Evans, 1937 & 5 & E \\
\hline Afrogegenes hottentota (Latreille, 1824) & Weymer, 1901 & 5 & C,D \\
\hline $\begin{array}{l}\text { Afrogegenes letterstedti (Wallengren, } \\
\text { 1857) }\end{array}$ & Evans, 1937 & 5 & $\mathrm{D}$ \\
\hline Gegenes pumilio gambica (Mabille, 1878) & M \& BS, 2009 & 5 & $\mathrm{D}$ \\
\hline \multicolumn{4}{|c|}{ PAPILIONIDAE } \\
\hline Papilio a. antimachus (Drury, 1782) & Carvalho, 1962 & 5 & A \\
\hline Papilio zalmoxis (Hewitson, 1864) & BS, 1983 & 5 & A \\
\hline Papilio bacelarae (BS \& M., 2009) & BS \& M, $2009(*)$ & 1,5 & A \\
\hline Papilio f. filaprae (Suffert, 1904) & Druce, 1875 , as $P$. cypraeophila & 5 & A \\
\hline Papilio m. mechowi (Dewitz, 1881) & Dewitz, $1881(*)$ & 5 & A \\
\hline Papilio mechowianus (Dewitz, 1885) & Aurivillius, 1928 & 6 & A \\
\hline Papilio zenobia (Fabricius, 1775) & BS \& Fernandes, 1966 & 5 & A \\
\hline Papilio cynorta (Fabricius, 1793) & Druce, 1875 & 5 & A \\
\hline $\begin{array}{l}\text { Papilio echerioides homeyeri (Plötz, } \\
1880 \text { ) }\end{array}$ & Plötz, 1880 (*) & 5 & $\mathrm{~A}, \mathrm{C}$ \\
\hline
\end{tabular}




\begin{tabular}{|c|c|c|c|}
\hline Taxon & First reference for Angola & V & $\mathrm{H}$ \\
\hline $\begin{array}{l}\text { Papilio chitondensis (BS \& Fernandes, } \\
\text { 1966) }\end{array}$ & BS \& Fernandes, $1966(*)$ & 1,5 & $\mathrm{~B}, \mathrm{C}$ \\
\hline $\begin{array}{l}\text { Papilio chrapkowskoides nurettini } \\
\text { (Koçak, 1983) }\end{array}$ & Bacelar, 1956, as $P$. bromius & 5 & A \\
\hline Papilio n. nireus (Linnaeus, 1758) & Druce, 1875 & 5 & $\mathrm{H}$ \\
\hline Papilio nireus lyaeus (Doubleday, 1845) & Ladeiro, 1956 & 5 & $\mathrm{~A}, \mathrm{D}$ \\
\hline Papilio sosia pulchra (Berger, 1950) & BS \& Fernandes, 1966 & 5 & A \\
\hline $\begin{array}{l}\text { Papilio mackinnoni benguellae (Jordan, } \\
\text { 1908) }\end{array}$ & Jordan, $1908(*)$ & 1,5 & A \\
\hline Papilio d. dardanus (Brown, 1776) & Druce, 1875 & 5 & $\mathrm{C}$ \\
\hline $\begin{array}{l}\text { Papilio phorcas congoanus (Rothschild, } \\
1896 \text { ) }\end{array}$ & BS \& Fernandes, 1964 & 5 & A \\
\hline Papilio h. hesperus (Westwood, 1843) & Aurivillius, 1928 & 5 & $\mathrm{~A}$ \\
\hline Papilio l. lormieri (Distant, 1874) & Bacelar, 1956 & 5 & A \\
\hline Papilio d. demodocus (Esper, 1798) & Weymer, 1901 & 5 & $\mathrm{H}$ \\
\hline Graphium a. angolanus (Goeze, 1779) & Goeze, $1779(*)$ & 5 & $\mathrm{H}$ \\
\hline Graphium schaffgotschi (Niepelt, 1927) & Villiers, 1979 & 4,5 & $\mathrm{D}$ \\
\hline Graphium ridleyanus (White, 1843) & Druce, 1875 & 5 & A \\
\hline $\begin{array}{l}\text { Graphium latreillianus theorini } \\
\text { (Aurivillius,1881) }\end{array}$ & Aurivillius, 1928 & 5 & A \\
\hline Graphium tynderaeus (Fabricius, 1793) & BS \& Fernandes, 1964 & 5 & A \\
\hline Graphium a. almansor (Honrath, 1884) & Aurivillius, 1928 & 5 & $\mathrm{~A}, \mathrm{C}$ \\
\hline $\begin{array}{l}\text { Graphium ucalegonides (Staudinger, } \\
\text { 1884) }\end{array}$ & Smith \& Vane-Wright, 2001 & 2 & A \\
\hline Graphium h. hachei (Dewitz, 1881) & Dewitz, $1881(*)$ & 5 & A \\
\hline Graphium poggianus (Honrath, 1884) & Aurivillius, 1928 & 3 & A \\
\hline Graphium u. ucalegon (Hewitson, 1865) & Bacelar, 1956 & 5 & A \\
\hline Graphium l. leonidas (Fabricius, 1793) & Druce, 1875 & 5 & $\mathrm{H}$ \\
\hline Graphium antheus (Cramer, 1779) & Druce, 1875 & 5 & A,D \\
\hline Graphium p. policenes (Cramer, 1775) & Druce, 1875 & 5 & $\mathrm{~A}, \mathrm{C}$ \\
\hline Graphium p. porthaon (Hewitson, 1865) & Gardiner, 2004 & 6 & $\mathrm{~B}, \mathrm{D}$ \\
\hline \multicolumn{4}{|l|}{ Pieridae | Pseudopontinae } \\
\hline $\begin{array}{l}\text { Pseudopontia paradoxa (Felder \& Felder, } \\
\text { 1869) }\end{array}$ & $\boldsymbol{\square}$ & 5 & A \\
\hline Pseudopontia australis (Dixey, 1923) & Snellen, 1882 , as $P$. paradoxa & 3,5 & A \\
\hline \multicolumn{4}{|l|}{ PiEridae | Coliadinae } \\
\hline Catopsilia florella (Fabricius, 1775) & Weymer, 1901 & 5 & $\mathrm{H}$ \\
\hline Colias e. electo (Linnaeus, 1763) & Gardiner, 2004 & 6 & $\mathrm{D}$ \\
\hline Colias electo hecate (Strecker, 1905) & Bacelar, 1948 & 5 & $\mathrm{~F}$ \\
\hline Eurema b. brigitta (Stoll, 1780) & Butler, 1871 & 5 & $\mathrm{D}, \mathrm{H}$ \\
\hline $\begin{array}{l}\text { Eurema desjardinsi regularis (Butler, } \\
\text { 1876) }\end{array}$ & Mabille, 1877 & 5 & $\mathrm{D}, \mathrm{C}$ \\
\hline Eurema floricola leonis (Butler, 1886) & Trimen, 1891, as E. floricola & 6 & B \\
\hline Eurema hapale (Mabille, 1882) & Ladeiro, 1956 & 5 & $\mathrm{~A}, \mathrm{~B}$ \\
\hline Eurema hecabe solifera (Butler, 1875) & Butler, $1875(*)$ & 5 & $\mathrm{D}$ \\
\hline
\end{tabular}




\begin{tabular}{|c|c|c|c|}
\hline Taxon & First reference for Angola & V & $\mathrm{H}$ \\
\hline Eurema senegalensis (Boisduval, 1836) & Butler, 1871 & 5 & A \\
\hline \multicolumn{4}{|l|}{ Pieridae | Pierinae } \\
\hline Pinacopteryx e. eriphia (Godart, 1819) & Butler, 1871 & 5 & B,D \\
\hline Nepheronia a. argia (Fabricius, 1775) & Druce, 1875 & 5 & A \\
\hline Nepheronia b. buquetii (Boisduval, 1836) & Druce, 1875 & 5 & $\mathrm{~B}, \mathrm{D}$ \\
\hline Nepheronia p.pharis (Boisduval, 1836) & Aurivillius, 1928 & 5 & $\mathrm{~B}$ \\
\hline $\begin{array}{l}\text { Nepheronia thalassina verulanus (Ward, } \\
\text { 1871) }\end{array}$ & Bacelar, 1958a, b & 5 & B \\
\hline Eronia cleodora Hübner, 1823 & Aurivillius, 1928 & 6 & $\mathrm{D}$ \\
\hline Afrodryas leda (Boisduval, 1847) & Bacelar, 1961 & 5 & $\mathrm{~B}$ \\
\hline Teracolus a. agoye (Wallengren, 1857) & Weymer, 1901 & 5 & $\mathrm{D}$ \\
\hline $\begin{array}{l}\text { Colotis calais williami (Henning \& } \\
\text { Henning, 1994) }\end{array}$ & Willis, 2009 & 6 & $\mathrm{D}$ \\
\hline $\begin{array}{l}\text { Colotis antevippe gavisa (Wallengren, } \\
\text { 1857) }\end{array}$ & Trimen, 1891 & 5 & $\mathrm{D}$ \\
\hline $\begin{array}{l}\text { Colotis celimene pholoe (Wallengren, } \\
1860 \text { ) }\end{array}$ & Talbot, 1939 & 4,5 & $\mathrm{~F}$ \\
\hline Colotis annae walkeri (Butler, 1884) & Butler, $1884(*)$ & 4,5 & $\mathrm{~F}$ \\
\hline Colotis doubledayi (Hopffer, 1862) & Hopffer, $1862(*)$ & 5 & $\mathrm{D}$ \\
\hline Colotis e. euippe (Linnaeus, 1758) & Latreille \& Godart, 1819 & 5 & $\mathrm{~B}$ \\
\hline Colotis euippe mediata (Talbot, 1939) & Talbot, 1939 & 5 & C,D \\
\hline $\begin{array}{l}\text { Colotis evagore antigone (Boisduval, } \\
\text { 1836) }\end{array}$ & Druce, 1875 & 5 & B \\
\hline Colotis e. evenina (Wallengren, 1857) & Trimen, 1891 & 5 & $\mathrm{~F}$ \\
\hline Colotis ione (Godart, 1819) & Bacelar, 1961 & 6 & $\mathrm{D}$ \\
\hline Colotis regina (Trimen, 1863 ) & Trimen, 1891 & 5 & $\mathrm{D}$ \\
\hline Colotis vesta rhodesinus (Butler, 1894) & Bacelar, 1958a, b & 5 & $\mathrm{D}$ \\
\hline Teracolus e. eris (Klug, 1829) & Druce, 1875 & 5 & $\mathrm{D}$ \\
\hline Teracolus subfasciatus (Swainson, 1833) & Aurivillius, 1928 & 5 & $\mathrm{C}$ \\
\hline Belenois aurota (Fabricius, 1793) & Trimen, 1891 & 5 & $\mathrm{H}$ \\
\hline Belenois calypso dentigera (Butler, 1888) & Druce, 1875 , as $B$. calypso & 5 & A \\
\hline $\begin{array}{l}\text { Belenois welwitschii welwitschii } \\
\text { (Rogenhofer, 1890) }\end{array}$ & Rogenhofer, $1890(*)$ & 5 & $\mathrm{C}$ \\
\hline Belenois crawshayi (Butler, 1894) & Aurivillius, 1928 & 5 & C,D \\
\hline Belenois creona severina $($ Stoll, 1781$)$ & Butler, 1871 & 5 & $\mathrm{H}$ \\
\hline Belenois g. gidica (Godart, 1819) & $\Delta$ & 5 & $\mathrm{D}$ \\
\hline Belenois $r$. rubrosignata (Weymer, 1901) & Weymer, $1901(*)$ & 3,5 & $\mathrm{C}$ \\
\hline Belenois s. solilucis (Butler, 1874) & Butler, $1874(*)$ & 5 & A \\
\hline $\begin{array}{l}\text { Belenois sudanensis mayumbana (Berger, } \\
\text { 1981) }\end{array}$ & 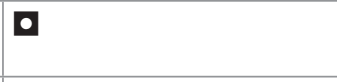 & 2,5 & $\mathbf{A}$ \\
\hline $\begin{array}{l}\text { Belenois sudanensis pseudodentigera } \\
\text { (Berger, 1981) }\end{array}$ & $\boldsymbol{0}$ & 5 & $\mathbf{C}$ \\
\hline Belenois theuszi (Dewitz, 1889) & Dewitz, $1889(*)$ & 5 & A \\
\hline \multicolumn{4}{|l|}{ Pieridae | Pierinae } \\
\hline Belenois t. thysa (Hopffer, 1855) & 口 & 5 & $\mathrm{C}$ \\
\hline
\end{tabular}




\begin{tabular}{|c|c|c|c|}
\hline Taxon & First reference for Angola & V & $\mathrm{H}$ \\
\hline Belenois thysa meldolae (Butler, 1872) & Butler, $1872(*)$ & 5 & A,B \\
\hline $\begin{array}{l}\text { Dixeia capricornus falkensteinii (Dewitz, } \\
\text { 1879) }\end{array}$ & Dewitz, $1879(*)$ & 2,5 & $\mathrm{~B}, \mathrm{C}$ \\
\hline Dixeia sp. & - + 2 + & 5 & A \\
\hline Dixeia pigea (Boisduval, 1836) & Aurivillius, 1928 & 5 & $\mathrm{C}$ \\
\hline Pontia h. helice (Linnaeus, 1764) & Willis, 2009 & 5 & $\mathrm{D}$ \\
\hline $\begin{array}{l}\text { Appias epaphia angolensis (M \& BS, } \\
\text { 2006) }\end{array}$ & M \& BS, $2006(*)$ & 1,5 & A,B \\
\hline Appias perlucens (Butler, 1898) & Butler, $1898(*)$ & 5 & A \\
\hline Appias phaola uigensis (M \& BS, 2006) & M \& BS, $2006(*)$ & 1,5 & A \\
\hline Appias s. sabina (Felder \& Felder, 1865) & Druce, 1875 & 5 & A \\
\hline Appias sylvia nyassana (Butler, 1897) & Druce, 1875 , as Belenois & 6 & A? \\
\hline Appias sylvia ribeiroi (M \& BS, 2006) & M \& BS, $2006(*)$ & 1,5 & A \\
\hline Leptosia a. alcesta (Stoll, 1782) & Druce, 1875 & 5 & A \\
\hline Leptosia h. hybrida (Bernardi, 1952) & 0 & 5 & $\mathrm{~A}, \mathrm{C}$ \\
\hline Leptosia n. nupta (Butler, 1873) & Butler, $1873(*)$ & 5 & A \\
\hline $\begin{array}{l}\text { Leptosia wigginsi pseudalcesta (Bernardi, } \\
\text { 1965) }\end{array}$ & - & 5 & A \\
\hline Mylothris carvalhoi (M \& BS, 2009) & M \& BS, $2009(*)$ & 1,5 & A \\
\hline $\begin{array}{l}\text { Mylothris mavunda (Hancock \& Heath, } \\
\text { 1985) }\end{array}$ & Koçak \& Kemal, 2009 & 3 & A? \\
\hline Mylothris a. agathina (Cramer, 1779) & Trimen, 1891 & 5 & $\mathrm{C}$ \\
\hline Mylothris asphodelus (Butler, 1888) & Aurivillius, 1928 & 5 & A \\
\hline Mylothris elodina diva (Berger, 1954) & Berger, 1981 & 2,5 & $\mathbf{C}$ \\
\hline Mylothris poppea (Cramer, 1777) & Druce, 1875 & 5 & A \\
\hline Mylothris rembina (Plötz, 1880) & Talbot, 1944 & 6 & A \\
\hline Mylothris rhodope (Fabricius, 1775) & Talbot, 1944 & 5 & $\mathrm{D}$ \\
\hline $\begin{array}{l}\text { Mylothris rueppellii rhodesiana (Riley, } \\
\text { 1921) }\end{array}$ & Talbot, 1944 & 5 & $\mathrm{C}$ \\
\hline Mylothris spica gabela (Berger, 1979) & Berger, $1979(*)$ & 1,6 & A \\
\hline Mylothris sulphurea (Aurivillius, 1895) & - & 5 & A \\
\hline Mylothris y. cf. yulei (Butler, 1897) & 口 & 5 & A \\
\hline \multicolumn{4}{|l|}{ LYCAENIDAE | Miletinae } \\
\hline Euliphyra mirifica (Holland, 1890) & Larsen, 2005 & 6 & A \\
\hline Aslauga m. marshalli (Butler, 1899) & Larsen, 2005 & 6 & A \\
\hline Megalopaplpus zymna (Westwood, 1851) & Ackery et al., 1995 & 5 & A \\
\hline Spalgis l. lemolea $($ Druce, 1890$)$ & Ladeiro, 1956 & 5 & $\mathrm{~A}, \mathrm{C}$ \\
\hline Lachnocnema angolanus (Libert, 1996) & Libert, 1996 b (*) & 5 & $\mathrm{~A}, \mathrm{D}$ \\
\hline Lachnocnema bamptoni (Libert, 1996) & Libert, 1996 b (*) & 6 & $\mathrm{C}$ \\
\hline Lachnocnema bibulus (Fabricius, 1793) & Libert, 1996 b & 5 & A,C,D \\
\hline Lachnocnema emperamus (Snellen, 1872) & Snellen, 1872 & 5 & A \\
\hline Lachnocnema intermedia (Libert, 1996) & Ladeiro, 1956 , as L. durbani $(*)$ & 5 & $\mathrm{C}$ \\
\hline Lachnocnema laches (Fabricius, 1793) & Libert, 1996 a & 5 & $\mathrm{~A}, \mathrm{C}$ \\
\hline Lachnocnema r. regularis (Libert, 1996) & Libert, $1996 \mathrm{c}$ & 6 & C? \\
\hline
\end{tabular}




\begin{tabular}{|c|c|c|c|}
\hline Taxon & First reference for Angola & $\mathrm{V}$ & $\mathrm{H}$ \\
\hline \multicolumn{4}{|l|}{ LyCAEnIDAe | Poritiinae } \\
\hline $\begin{array}{l}\text { Alaena amazoula congoana (Aurivillius, } \\
\text { 1914) }\end{array}$ & Aurivillius, $1914(*)$ & 6 & G \\
\hline Alaena rosei (Vane-Wright, 1980) & Vane-Wright, $1980(*)$ & 1,5 & G \\
\hline Pentila maculata pardalena (Druce, 1910) & Stempffer \& Bennett, 1961 & 6 & A \\
\hline Pentila amenaida (Hewitson, 1873) & Hewitson, $1873(*)$ & 5 & A \\
\hline $\begin{array}{l}\text { Pentila pauli benguellana (Stempffer \& } \\
\text { Bennett, 1961) }\end{array}$ & Stempffer \& Bennett, $\left.1961{ }^{*}\right)$ & 5 & $\mathrm{~A}, \mathrm{~B}$ \\
\hline Pentila t. tachyroides (Dewitz, 1879) & Dewitz, $1879(*)$ & 5 & A \\
\hline $\begin{array}{l}\text { Telipna acraeoides (Grose-Smith \& } \\
\text { Kirby, 1890) }\end{array}$ & Grose-Smith \& Kirby, $1890(*)$ & 5 & A \\
\hline Telipna a. albofasciata (Aurivillius, 1910) & Libert, 2005 & 6 & A \\
\hline Telipna atrinervis (Hulstaert, 1924) & 口 & 5 & A \\
\hline Telipna cuypersi (Libert, 2005) & Libert, 2005 & 6 & A \\
\hline $\begin{array}{l}\text { Telipna nyanza katangae (Stempffer, } \\
\text { 1961) }\end{array}$ & Libert, 2005 & 6 & A \\
\hline Telipna s. sanguinea (Plötz, 1880) & Aurivillius, 1928 & 5 & A \\
\hline $\begin{array}{l}\text { Ornipholidotus gabonensis (Stempffer, } \\
\text { 1947) }\end{array}$ & $\Delta$ & 6 & A \\
\hline $\begin{array}{l}\text { Ornipholidotus perfragilis (Holland, } \\
1890 \text { ) }\end{array}$ & Libert, 2005 & 6 & A \\
\hline $\begin{array}{l}\text { Ornipholidotus ugandae goodi (Libert, } \\
2000 \text { ) }\end{array}$ & Libert, 2005 & 6 & $\mathrm{~A}$ \\
\hline Cooksonia nozolinoi (M \& BS, 2007) & M \& BS, $2007(*)$ & 1,5 & $\mathrm{C}$ \\
\hline $\begin{array}{l}\text { Mimacraea charmian (Grose-Smith \& } \\
\text { Kirby, 1890) }\end{array}$ & Grose-Smith \& Kirby, $1890(*)$ & 6 & A \\
\hline Mimacraea landbecki (Druce, 1910) & Libert, $2000 \mathrm{~b}$ & 5 & A \\
\hline Mimacraea marshalli (Trimen, 1898) & Libert, $2000 \mathrm{~b}$ & 5 & A \\
\hline $\begin{array}{l}\text { Mimeresia debora deborula (Aurivillius, } \\
\text { 1899) }\end{array}$ & - & 5 & A \\
\hline Eresiomera osheba (Holland, 1890) & $\Delta$ & 5 & A \\
\hline Citrinophila e. erastus (Hewitson, 1866) & Aurivillius, 1928 & 6 & $\mathrm{~A}$ \\
\hline $\begin{array}{l}\text { Cnodontes vansomereni (Stempffer \& } \\
\text { Bennett, 1953) }\end{array}$ & $\Delta$ & 5 & $\mathrm{D}$ \\
\hline Liptena evanescens (Kirby, 1887) & - & 5 & A \\
\hline Liptena fatima (Kirby, 1890) & - & 5 & A \\
\hline Liptena h. homeyeri (Dewitz, 1884) & $\Delta$ & 6 & A \\
\hline $\begin{array}{l}\text { Liptena homeyeri straminea (Stempffer, } \\
\text { Bennett \& May, 1974) }\end{array}$ & Stempffer, Bennett \& May, 1974 (*) & 1,6 & A \\
\hline Liptena parva (Kirby, 1887) & - & 5 & $\mathrm{~A}$ \\
\hline Liptena undularis (Hewitson, 1866) & Druce, 1875 & 5 & A \\
\hline $\begin{array}{l}\text { Liptena xanthostola xantha (Grose-Smith, } \\
\text { 1901) }\end{array}$ & Larsen, 2005 & 6 & A \\
\hline Falcuna h. hollandii (Aurivillius, 1895) & Ackery et al., 1995 & 6 & A \\
\hline $\begin{array}{l}\text { Falcuna lacteata (Stempffer \& Bennett, } \\
\text { 1963) }\end{array}$ & Stempffer \& Bennett, $1963(*)$ & 1,6 & A \\
\hline
\end{tabular}




\begin{tabular}{|c|c|c|c|}
\hline Taxon & First reference for Angola & $\mathrm{V}$ & $\mathrm{H}$ \\
\hline $\begin{array}{l}\text { Falcuna libyssa angolensis (Stempffer \& } \\
\text { Bennett, 1963) }\end{array}$ & Stempffer \& Bennett, 1963 (*) $^{*}$ & 1,5 & A \\
\hline Falcuna s. synesia (Hulstaert, 1924) & Stempffer \& Bennett, $1963(*)$ & 2 & A \\
\hline $\begin{array}{l}\text { Tetrarhanis ilala etoumbi (Stempffer, } \\
\text { 1964) }\end{array}$ & - & 5 & A \\
\hline Tetrarhanis i. ilma (Hewitson, 1873) & Hewitson, $1873(*)$ & 6 & A \\
\hline Larinopoda lircaea (Hewitson, 1866) & Stempffer, 1957 & 6 & A \\
\hline Larinopoda tera (Hewitson, 1873) & Aurivillius, 1928 & 5 & A \\
\hline $\begin{array}{l}\text { Hewitsonia bitjeana (Bethune-Baker, } \\
\text { 1915) }\end{array}$ & - & 5 & A \\
\hline Hewitsonia k. kirbyi (Dewitz, 1879) & Dewitz, $1879(*)$ & 6 & A \\
\hline Cerautola ceraunia (Hewitson, 1873) & Larsen, 2005 & 6 & A \\
\hline $\begin{array}{l}\text { Cerautola crowleyi leucographa (Libert, } \\
\text { 1999) }\end{array}$ & Libert, 1999 & 6 & A \\
\hline Hewitola hewitsonii (Mabille, 1877) & Mabille, $1877(*)$ & 6 & A \\
\hline Cerautola miranda vidua (Talbot, 1935) & Bacelar, 1958a, b & 5 & A \\
\hline Epitola posthumus (Fabricius, 1793) & Bacelar, 1956 & 5 & A \\
\hline Epitola urania $($ Kirby, 1887$)$ & Libert, 1999 & 6 & $\mathbf{A}$ \\
\hline Hypophytala h. hyetta (Hewitson, 1873) & Hewitson, $1873(*)$ & 2 & $\mathbf{A}$ \\
\hline Stempfferia cercene (Hewitson, 1873) & Hewitson, $1873(*)$ & 6 & $\mathbf{A}$ \\
\hline Stempfferia cinerea (Berger, 1981) & Libert, 1999 & 2 & A \\
\hline $\begin{array}{l}\text { Stempfferia michelae centralis (Libert, } \\
\text { 1999) }\end{array}$ & Libert, 1999 & 5 & A \\
\hline Deloneura barca (Grose-Smith, 1901) & Grose-Smith, $1901\left(^{*}\right)$ & 1,6 & C?,D? \\
\hline $\begin{array}{l}\text { Deloneura cf. subfusca (Hawker-Smith, } \\
\text { 1933) }\end{array}$ & - & 5 & $\mathrm{~F}$ \\
\hline Epitolina dispar (Kirby, 1887) & Larsen, 2005 & 6 & A \\
\hline Epitolina melissa (Druce, 1888) & Larsen, 2005 & 6 & A \\
\hline \multicolumn{4}{|l|}{ LYCAENIDAE | Theclinae } \\
\hline Myrina s. silenus (Fabricius, 1775) & Druce, 1875 & 5 & $\mathrm{~B}, \mathrm{D}$ \\
\hline Myrina silenus ficedula (Trimen, 1879) & Gardiner, 2004 & 6 & $\mathrm{D}$ \\
\hline Oxylides binza (Berger, 1981) & Druce, 1875 as $O$. faunus & 2,5 & A \\
\hline \multicolumn{4}{|l|}{ LYCAENIDAE | Theclinae } \\
\hline $\begin{array}{l}\text { Oxylides feminina stempfferi (Berger, } \\
\text { 1981) }\end{array}$ & Libert, 2004 & 6 & A \\
\hline Syrmoptera amasa (Hewitson, 1869) & Libert, 2004 & 6 & A \\
\hline Syrmoptera homeyerii (Dewitz, 1879) & Dewitz, $1879(*)$ & 6 & A \\
\hline $\begin{array}{l}\text { Dapidodigma demeter nuptus (Clench, } \\
\text { 1961) }\end{array}$ & Larsen, 2005 & 3,5 & A \\
\hline \multicolumn{4}{|l|}{ LYCAENIDAE | Aphnaeinae } \\
\hline Lipaphneus a. cf. aderna (Plötz, 1880) & • & 5 & A \\
\hline Crudaria leroma (Wallengren, 1857) & Gardiner, 2004 & 6 & $\mathrm{D}$ \\
\hline $\begin{array}{l}\text { Aloeides angolensis (Tite \& Dickson, } \\
\text { 1973) }\end{array}$ & Tite \& Dickson, $1973(*)$ & 1,6 & $\mathrm{~F}$ \\
\hline Aphnaeus erikssoni (Trimen, 1891) & Trimen, $1891(*)$ & 5 & $\mathrm{C}$ \\
\hline
\end{tabular}




\begin{tabular}{|c|c|c|c|}
\hline Taxon & First reference for Angola & $\mathrm{V}$ & $\mathrm{H}$ \\
\hline Aphnaeus orcas (Drury, 1782) & Larsen, 2005 & 6 & A \\
\hline Aphnaeus affinis (Riley, 1921) & Libert, 2013 & & \\
\hline Erikssonia acraeina (Trimen, 1891) & Trimen, $1891(*)$ & 6 & $\mathrm{D}$ \\
\hline Pseudaletis a. agrippina (Druce, 1888) & $\Delta$ & 5 & A \\
\hline Cigaritis ella (Hewitson, 1865) & Gardiner, 2004 & 6 & $\mathrm{D}$ \\
\hline Cigaritis phanes (Trimen, 1873) & Weymer, 1901 & 6 & $\mathrm{~F}$ \\
\hline Cigaritis homeyeri (Dewitz, 1887) & Aurivillius, 1928 & 5 & $\mathrm{C}$ \\
\hline Cigaritis m. modestus (Trimen, 1891) & Trimen, $1891(*)$ & $1,4,5$ & $\mathrm{~A}, \mathrm{C}$ \\
\hline Cigaritis mozambica (Bertoloni, 1850) & $\mathbf{\Delta}$ & 6 & $\mathrm{D}$ \\
\hline Cigaritis natalensis (Westwood, 1851) & Ladeiro, 1956 & 6 & C,D \\
\hline $\begin{array}{l}\text { Cigaritis trimeni congolanus (Dufrane, } \\
\text { 1954) }\end{array}$ & $\Delta$ & 2,5 & A \\
\hline Zeritis fontainei (Stempffer, 1956) & Willis, 2009 & 6 & $\mathrm{C}, \mathrm{G}$ ? \\
\hline Zeritis krystyna (D’Abrera, 1980) & D’Abrera, 1980 (*) & 1,6 & C? \\
\hline Zeritis sorhagenii (Dewitz, 1879) & Dewitz, $1879(*)$ & 6 & $\mathrm{C}$ ? \\
\hline Axiocerces $a$. amanga (Westwood, 1881) & Trimen, 1891 & 5 & $\mathrm{C}$ \\
\hline $\begin{array}{l}\text { Axiocerces bambana orichalcea (Henning } \\
\& \text { Henning, 1996) }\end{array}$ & • & 5 & B \\
\hline $\begin{array}{l}\text { Axiocerces amanga baumi (Weymer, } \\
\text { 1901) }\end{array}$ & Weymer, $1901(*)$ &, 5 & $\mathrm{C}$ \\
\hline Axiocerces t. tjoanae (Wallengren, 1857) & Henning \& Henning, 1996 & 6 & $\mathrm{~B}$ \\
\hline $\begin{array}{l}\text { Iolaus hemicyanus barnsi (Joicey \& } \\
\text { Talbot, 1921) }\end{array}$ & - & 2,5 & A \\
\hline Iolaus i. iasis (Hewitson, 1865) & Larsen, 2005 & 6 & A \\
\hline $\begin{array}{l}\text { Iolaus mimosae rhodosense (Stempffer \& } \\
\text { Bennett, 1959) }\end{array}$ & Gardiner, 2004 & 6 & $\mathrm{D}$ \\
\hline Iolaus obscura (Aurivillius, 1923) & - & 4,5 & $\mathrm{D}$ \\
\hline Iolaus violacea (Riley, 1928) & Riley, 1928 (*) & 5 & $\mathrm{C}$ \\
\hline Iolaus pallene (Wallengren, 1857) & Gardiner, 2004 & 6 & $\mathrm{D}$ \\
\hline Iolaus trimeni (Wallengren, 1875) & Ackery et al., 1995 & 5 & C,D \\
\hline Iolaus iturensis (Joicey \& Talbot, 1921) & $\Delta$ & 6 & $\mathrm{C}$ \\
\hline Iolaus parasilanus mabillei (Riley, 1928) & Riley, $1928(*)$ & 2 & A \\
\hline Iolaus s. silarus (Druce, 1885$)$ & Gardiner, 2004 & 5 & C,D \\
\hline Iolaus t. timon (Fabricius, 1787) & Ackery et al., 1995 & 6 & A \\
\hline Hemiolaus vividus (Pinhey, 1962) & Aurivillius, 1928 , as caeculus & 5 & $\mathrm{C}, \mathrm{D}$ \\
\hline Stugeta bowkeri maria (Suffert, 1904) & Druce, 1875 , as bowkeri & 3,5 & $\mathrm{~B}$ \\
\hline Stugeta bowkeri tearei (Dikson, 1980) & Gardiner, 2004 & 6 & A,C,E \\
\hline $\begin{array}{l}\text { Hypolycaena a. antifaunus (Westwood, } \\
\text { 1851) }\end{array}$ & Druce, 1875 & 5 & A \\
\hline Hypolycaena h. hatita (Hewitson, 1865) & Druce, 1875 & 5 & A \\
\hline Hypolycaena l. lebona (Hewitson, 1865) & Druce, 1875 & 6 & A \\
\hline Hypolycaena naara (Hewitson, 1873) & Hewitson, $1873(*)$ & 6 & A \\
\hline $\begin{array}{l}\text { Hypolycaena nigra (Bethune-Baker, } \\
\text { 1914) }\end{array}$ & M \& BS, 2012 & 5 & A \\
\hline
\end{tabular}




\begin{tabular}{|c|c|c|c|}
\hline Taxon & First reference for Angola & $\mathrm{V}$ & $\mathrm{H}$ \\
\hline $\begin{array}{l}\text { Hypolycaena p. philippus (Fabricius, } \\
\text { 1793) }\end{array}$ & Druce, 1875 & 5 & $\mathrm{D}$ \\
\hline $\begin{array}{l}\text { Hypolycaena buxtoni spurcus (Talbot, } \\
1929 \text { M \& BS., 2012) }\end{array}$ & M \& BS, 2012 & 5 & F? \\
\hline Pilodeudorix badhami (Carcasson, 1961) & Libert, 2004 & 6 & $?$ \\
\hline Pilodeudorix caerulea (Druce, 1890) & Libert, 2004 & 5 & C,D \\
\hline $\begin{array}{l}\text { Pilodeudorix pseudoderitas (Stempffer, } \\
\text { 1964) }\end{array}$ & Larsen, 2005 & 6 & A \\
\hline Pilodeudorix zeloides (Butler, 1901) & Libert, 2004 & 6 & $\mathrm{C}$ \\
\hline Paradeudorix cobaltina (Stempffer, 1964) & Larsen, 2005 & 6 & A \\
\hline $\begin{array}{l}\text { Leptomyrina henningi angolensis (M \& } \\
\text { BS, 2009) }\end{array}$ & M \& BS, $2009\left(^{*}\right)$ & 1,5 & B \\
\hline Pilodeudorix deritas (Hewitson, 1874) & Hewitson, $1874(*)$ & 5 & A \\
\hline Pilodeudorix m. mera (Hewitson, 1873) & Hewitson, $1873(*)$ & 5 & A \\
\hline $\begin{array}{l}\text { Pilodeudorix otraeda genuba (Hewitson, } \\
1875 \text { ) }\end{array}$ & $\Delta$ & 6 & A \\
\hline Hypomyrina nomenia (Hewitson, 1874) & Larsen, 2005 & 5 & A \\
\hline Deudorix antalus (Hopffer, 1855) & Bacelar, 1948 & 5 & $\mathrm{D}, \mathrm{G}$ \\
\hline Deudorix caliginosa (Lathy, 1903) & $\Delta$ & 6 & $\mathrm{C}$ \\
\hline Deudorix dinochares (Grose-Smith, 1887) & Gardiner, 2004 & 5 & C,D \\
\hline Deudorix cf. diocles (Hewitson, 1869) & Libert, 2004 & 5 & C \\
\hline Deudorix lorisona coffea (Jackson, 1966) & Libert, 2004 & 5 & $\mathrm{~A}, \mathrm{C}$ \\
\hline Capys c. connexiva (Butler, 1896) & Henning \& Henning, 1988 & 6 & $\mathrm{X}$ \\
\hline \multicolumn{4}{|l|}{ LyCAENIDAE | Polyommatine } \\
\hline Anthene akoae (Libert, 2010) & Libert. 2010 & 6 & $?$ \\
\hline Anthene alberta (Bethune-Baker, 1910) & Aurivillius, 1928 & 5 & $\mathrm{~A}, \mathrm{C}$ \\
\hline $\begin{array}{l}\text { Anthene a. amarah (Guérin-Méneville, } \\
\text { 1849) }\end{array}$ & Stempffer, 1957 & 5 & $\mathrm{D}$ \\
\hline Anthene lvida livida (Trimen, 1881) & Gardiner, 2004 & 6 & $\mathrm{D}$ \\
\hline Anthene c. crawshayi (Butler, 1899) & 口 & 5 & $\mathrm{D}$ \\
\hline Anthene d. definita (Butler, 1899) & Gardiner, 2004 & 5 & A \\
\hline Anthene larydas (Cramer, 1780) & Weymer, 1901 & 5 & A \\
\hline Anthene l. ligures (Hewitson, 1874) & Hewitson, $1874(*)$ & 6 & A \\
\hline Anthene liodes (Hewitson, 1874) & Aurivillius, 1909 & 6 & A,D \\
\hline Anthene l. lunulata (Trimen, 1894) & Trimen, $1894(*)$ & 5 & $\mathrm{D}$ \\
\hline $\begin{array}{l}\text { Anthene nigropunctata (Bethune-Baker, } \\
\text { 1910) }\end{array}$ & Gardiner, 2004 & 6 & $?$ \\
\hline Anthene princeps (Butler, 1876) & Gardiner, 2004 & 5 & A,D \\
\hline Anthene r. rubricinctus (Holland, 1891) & Aurivillius, 1928 & 6 & A \\
\hline Anthene sylvanus (Drury, 1773) & Aurivillius, 1928 & 6 & A \\
\hline Anthene talboti (Stempffer, 1936) & Libert, 2010 & 6 & $\mathrm{D}$ \\
\hline $\begin{array}{l}\text { Neurellipes flavomaculatus (Grose-Smith } \\
\& \text { Kirby, 1893) }\end{array}$ & Aurivillius, 1928 & 6 & A \\
\hline Neurellipes lachares (Hewitson, 1878) & Larsen, 2005 & 6 & A \\
\hline Neurellipes onias (Hulstaert, 1924) & Willis, 2009 & 6 & $?$ \\
\hline
\end{tabular}




\begin{tabular}{|c|c|c|c|}
\hline Taxon & First reference for Angola & V & $\mathrm{H}$ \\
\hline Neurellipes pyroptera (Aurivillius, 1895) & Libert, 2010 & 6 & A \\
\hline Neurypexina lyzanius (Hewitson, 1874) & Druce, 1875 & 6 & A \\
\hline Triclema lacides (Hewitson, 1874) & Hewitson, $1874(*)$ & 6 & A \\
\hline Triclema lucretilis (Hewitson, 1874) & Stempffer, 1957 & 6 & A \\
\hline Triclema cf. nigeriae (Aurivillius, 1905) & Libert, 2010 & 5 & $\mathrm{D}$ \\
\hline Monile g. gemmifera (Neave, 1910) & Libert, 2010 & 6 & A \\
\hline Cupidesthes vidua (Talbot, 1929) & Talbot, $1929(*)$ & 1,6 & $\mathrm{C}$ ? \\
\hline $\begin{array}{l}\text { Pseudonacaduba aethiops (Mabille, } \\
\text { 1877) }\end{array}$ & Mabille, $1877(*)$ & 5 & A \\
\hline $\begin{array}{l}\text { Pseudonacaduba s. sichela (Wallengren, } \\
\text { 1857) }\end{array}$ & Weymer, 1901 & 5 & $\mathrm{D}$ \\
\hline Lampides boeticus (Linnaeus, 1767) & Butler, 1871 & 5 & $\mathrm{H}$ \\
\hline $\begin{array}{l}\text { Uranothauma falkensteinii (Dewitz, } \\
\text { 1879) }\end{array}$ & Dewitz, 1879 (*) & 5 & A? \\
\hline $\begin{array}{l}\text { Uranothauma antinorii cf. felthami } \\
\text { (Stevenson, 1934) }\end{array}$ & BS \& M, 2007 & 5 & A \\
\hline $\begin{array}{l}\text { Uranothauma h. heritsia (Hewitson, } \\
\text { 1876) }\end{array}$ & Stempffer, 1957 & 5 & A,D \\
\hline Uranothauma nozolinoi (BS \& M., 2007) & BS \& M, $2007(*)$ & 1,5 & $\mathrm{C}$ \\
\hline Uranothauma poggei (Dewitz, 1879) & Dewitz, $1879(*)$ & 5 & A,C,D \\
\hline Uranothauma c. cyara (Hewitson, 1876) & Hewitson, $1876(*)$ & 5 & A? \\
\hline Cacyreus lingeus (Stoll, 1782) & Gardiner, 2004 & 5 & $\mathrm{~B}, \mathrm{D}$ \\
\hline Cacyreus marshalli (Butler, 1898) & Gardiner, 2004 & 6 & $\mathrm{D}$ \\
\hline Cacyreus virilis (Aurivillius, 1924) & Aurivillius, $1924(*)$ & 6 & $\mathrm{D}$ \\
\hline Leptotes babaulti (Stempffer, 1935) & Stempffer, 1957 & 5 & A,D \\
\hline Leptotes brevidentatus (Tite, 1958) & Tite, $1958(*-$ in part $)$ & 5 & $\mathrm{~A}, \mathrm{C}, \mathrm{D}$ \\
\hline Leptotes jeanneli (Stempffer, 1935) & Stempffer, 1957 & 5 & A,D \\
\hline Leptotes p. pirithous (Linnaeus, 1767) & Snellen, 1882 & 5 & $\mathrm{D}$ \\
\hline Leptotes p. pulchra (Murray, 1874) & Gardiner, 2004 & 5 & $\mathrm{E}$ \\
\hline Tuxentius calice (Hopffer, 1855) & Gardiner, 2004 & 5 & $\mathrm{E}$ \\
\hline Tuxentius c. carana (Hewitson, 1876) & Hewitson, $1876(*)$ & 5 & A \\
\hline Tuxentius margaritaceus (Sharpe, 1892) & Larsen, 2005 & 6 & A \\
\hline Tuxentius m. melaena (Trimen, 1887) & Aurivillius, 1928 & 6 & C,D \\
\hline $\begin{array}{l}\text { Tarucus sybaris linearis (Aurivillius, } \\
\text { 1924) }\end{array}$ & Aurivillius, 1928 & 6 & $\mathrm{D}$ \\
\hline Actizera lucida (Trimen, 1883) & Stempffer, 1957 & 5 & $\mathrm{~F}$ \\
\hline $\begin{array}{l}\text { Eicochrysops eicotrochilus (Bethune- } \\
\text { Baker, 1924) }\end{array}$ & $\Delta$ & 6 & $\mathrm{C}$ \\
\hline $\begin{array}{l}\text { Eicochrysops hippocrates (Fabricius, } \\
\text { 1793) }\end{array}$ & Gardiner, 2004 & 5 & $\mathrm{~A}, \mathrm{~B}, \mathrm{D}$ \\
\hline $\begin{array}{l}\text { Eicochrysops messapus mahallakoaena } \\
\text { (Wallengren, 1857) }\end{array}$ & Weymer, 1901, as messapus & 6 & $\mathrm{C}$ \\
\hline Cupidopsis c. cissus (Godart, 1824) & Ladeiro, 1956 & 5 & $\mathrm{E}$ \\
\hline Cupidopsis j. jobates (Hopffer, 1855) & Stempffer, 1957 & 5 & $\mathrm{D}$ \\
\hline Euchrysops barkeri (Trimen, 1893) & Aurivillius, 1928 & 6 & $\mathrm{C}$ \\
\hline
\end{tabular}




\begin{tabular}{|c|c|c|c|}
\hline Taxon & First reference for Angola & $\mathrm{V}$ & $\mathrm{H}$ \\
\hline Euchrysops malathana (Boisduval, 1833) & Stempffer, 1957 & 5 & C,D \\
\hline Euchrysops osiris (Hopffer, 1855) & Druce, 1875 & 5 & $\mathrm{C}, \mathrm{D}$ \\
\hline $\begin{array}{l}\text { Euchrysops subpallida (Bethune-Baker, } \\
\text { 1923) }\end{array}$ & Gardiner, 2004 & 6 & $\mathrm{~F}, \mathrm{G}$ \\
\hline \multicolumn{4}{|l|}{$\begin{array}{l}\text { Lepidochrysops abyssiniensis loveni } \\
\text { (Aurivillius, 1921) }\end{array}$} \\
\hline Lepidochrysops ansorgei (Tite, 1959) & Tite, $1959(*)$ & 1,5 & C,D \\
\hline $\begin{array}{l}\text { Lepidochrysops chlouages (Bethune- } \\
\text { Baker, 1923) }\end{array}$ & $\Delta$ & 5 & A,D \\
\hline $\begin{array}{l}\text { Lepidochrysops flavisquamosa (Tite, } \\
\text { 1959) }\end{array}$ & Tite, $1959(*)$ & 1,6 & $\mathrm{C}$ \\
\hline Lepidochrysops fulvescens (Tite, 1961) & Tite, $1961(*)$ & 1,6 & $\mathrm{C}$ \\
\hline Lepidochrysops g. glauca (Trimen, 1887) & Bacelar, 1948 & 5 & $\mathrm{D}$ \\
\hline Lepidochrysops hawkeri (Talbot, 1929) & Talbot, $1929(*)$ & 1,5 & C? \\
\hline Lepidochrysops nacrescens (Tite, 1961) & Tite, $1961(*)$ & 1,6 & $\mathrm{C}$ \\
\hline $\begin{array}{l}\text { Lepidochrysops reichenowi (Dewitz, } \\
\text { 1879) }\end{array}$ & Dewitz, $1879(*)$ & 1,6 & $?$ \\
\hline Thermoniphas distincta (Talbot, 1935) & 口 & 5 & $\mathrm{C}$ \\
\hline $\begin{array}{l}\text { Thermoniphas } p \text {. plurilimbata (Karsch, } \\
\text { 1895) }\end{array}$ & $\boldsymbol{\square}$ & 5 & A \\
\hline Thermoniphas t. togara (Plötz, 1880) & $\Delta$ & 6 & $\mathrm{~A}$ \\
\hline Oboronia guessfeldtii (Dewitz, 1879) & Larsen, 1991 & 5 & $\mathrm{~B}$ \\
\hline Oboronia pseudopunctatus (Strand, 1912) & $\Delta$ & 6 & A \\
\hline Oboronia punctatus (Dewitz, 1879) & & 5 & A \\
\hline Actizera lucida (Trimen, 1883) & Willis, 2009 & 6 & $\mathrm{C}$ \\
\hline Brephidium metophis (Wallengren, 1860) & Willis, 2009 & 6 & $?$ \\
\hline Azanus isis (Drury, 1773) & Bacelar, 1948 & 5 & A \\
\hline Azanus jesous (Guérin-Méneville, 1849) & Trimen, 1891 & 6 & $\mathrm{D}$ \\
\hline Azanus mirza (Plötz, 1880) & Stempffer, 1957 & 5 & $\mathrm{D}, \mathrm{A}$ \\
\hline Azanus moriqua (Wallengren, 1857) & Weymer, 1901 & 5 & $\mathrm{D}$ \\
\hline Azanus natalensis (Trimen, 1887) & Bacelar, 1948 & 5 & $\mathrm{D}$ \\
\hline Azanus ubaldus (Stoll, 1782) & Bacelar, 1948 & 5 & $\mathrm{~F}$ \\
\hline Chilades trochylus (Freyer, 1844) & Ladeiro, 1956 & 5 & $\mathrm{D}$ \\
\hline Zizeeria k. knysna (Trimen, 1862) & Ladeiro, 1956 & 5 & $\mathrm{D}$ \\
\hline Zizina otis antanossa (Mabille, 1877) & $\Delta$ & 5 & $\mathrm{D}$ \\
\hline Zizula hylax (Fabricius, 1775) & Gardiner, 2004 & 5 & $\mathrm{H}$ \\
\hline
\end{tabular}

\section{RIODINIDAE}

Afriodinia dewitzi (Aurivillius, 1899)

Afriodinia intermedia (Aurivillius, 1895)

Afriodinia r. rogersi (Druce, 1878)

Afriodinia tantalus caerulea (Riley, 1932)

Larsen, 2005

Druce, 1878 (*)

Druce, 1875 as tantalus

NympHaLIDAE | Libytheinae

\begin{tabular}{l|l|l|l}
\hline Libythea labdaca (Westwood, 1851) & Snellen, 1882 & 5 & A \\
\hline Libythea laius (Trimen, 1879) & Gardiner, 2004 & 5 & A \\
\hline
\end{tabular}




\begin{tabular}{|c|c|c|c|}
\hline Taxon & First reference for Angola & $\mathrm{V}$ & $\mathrm{H}$ \\
\hline \multicolumn{4}{|l|}{ NYMPHALIDAE | Danainae } \\
\hline Danaus c. orientis (Aurivillius, 1909) & Butler, 1871 & 5 & $\mathrm{H}$ \\
\hline Tirumala petiverana (Doubleday, 1847) & Butler, 1866 , as Danais leonora & 5 & $\mathrm{D}$ \\
\hline Amauris n. niavius (Linnaeus, 1758) & Aurivillius, 1928 & 5 & $\mathrm{~A}, \mathrm{~B}, \mathrm{D}$ \\
\hline Amauris t. tartarea (Mabille, 1876) & Mabille, $1876(*)$ & 5 & A \\
\hline $\begin{array}{l}\text { Amauris crawshayi angola (Bethune- } \\
\text { Baker, 1914) }\end{array}$ & Bethune-Baker, $1914(*)$ & 1,6 & A \\
\hline Amauris h. hecate (Butler, 1866) & - & 5 & A \\
\hline Amauris d. dannfelti (Aurivillius, 1891) & Aurivillius, $1891(*)$ & 1,5 & $\mathrm{~A}, \mathrm{C}$ \\
\hline Amauris h. hyalites (Butler, 1874) & Butler, $1874(*)$ & 5 & A \\
\hline Amauris vashti (Butler, 1869) & - & 5 & A \\
\hline \multicolumn{4}{|l|}{ NYMPHALIDAE | Satyrinae } \\
\hline $\begin{array}{l}\text { Gnophodes betsimena parmeno } \\
\text { (Doubleday, 1849) }\end{array}$ & Aurivillius, 1928 & 5 & A \\
\hline Gnophodes chelys (Fabicius, 1793) & Aurivillius, 1928 & 5 & A \\
\hline Melanitis leda (Linnaeus, 1758) & Bacelar, 1948 & 5 & $\mathrm{~B}, \mathrm{H}$ \\
\hline Elymnias b. bammakoo (Westwood, 1851) & Druce, 1875 & 5 & A \\
\hline Bicyclus iccius (Hewitson, 1865) & Larsen, 2005 & 6 & A \\
\hline Bicyclus sebetus (Hewitson, 1877) & Aurivillius, 1928 & 5 & A \\
\hline Bicyclus s. saussurei (Dewitz, 1879) & Dewitz, $1879(*)$ & 3,5 & A \\
\hline Bicyclus s. suffusa (Riley, 1921) & $\mathbf{\Delta}$ & 3,5 & $\mathrm{C}$ \\
\hline Bicyclus taenias (Hewitson, 1877) & - & 5 & A,B \\
\hline Bicyclus nachtetis (Condamin, 1965) & $\Delta$ & 3 & A \\
\hline Bicyclus technatis (Hewitson, 1877) & Larsen, 2005 & 6 & A \\
\hline Bicyclus vulgaris (Butler, 1868) & Druce, 1875 & 5 & A,D \\
\hline Bicyclus moyses (Condamin \& Fox, 1964) & Condamin \& Fox, 1964 & 5 & A \\
\hline Bicyclus sandace (Hewitson, 1877) & Bacelar, 1958a, b & 5 & A,D \\
\hline Bicyclus auricruda fulgida (Fox, 1963) & Aurivillius, 1928, as auricruda & 5 & A \\
\hline $\begin{array}{l}\text { Bicyclus collinsi (Aduse-Poku et al., } \\
\text { 2009) }\end{array}$ & Hewitson, 1873 & 5 & $\mathrm{~B}, \mathrm{D}$ \\
\hline Bicyclus angulosa selousi (Trimen, 1895) & Condamin, 1963 & 5 & $\mathrm{C}, \mathrm{D}$ \\
\hline Bicyclus campus (Karsch, 1893) & $\mathbf{\Delta}$ & 6 & A \\
\hline Bicyclus a. anynana (Butler, 1879) & Gardiner, 2004 & 6 & $\mathrm{D}$ \\
\hline $\begin{array}{l}\text { Bicyclus anynana centralis (Condamin, } \\
\text { 1968) }\end{array}$ & Condamin, 1968 & 5 & A \\
\hline Bicyclus cottrelli (van Son, 1952) & $\mathbf{\Delta}$ & 5 & $\mathrm{C}$ \\
\hline Bicyclus s. safitza (Westwood, 1850) & Butler, 1871, as Mycalesis caffra & 5 & $\mathrm{D}$ \\
\hline $\begin{array}{l}\text { Bicyclus funebris (Guérin-Méneville, } \\
\text { 1844) }\end{array}$ & Ladeiro, 1956 & 5 & $\mathrm{~A}, \mathrm{~B}, \mathrm{D}$ \\
\hline Bicyclus istaris (Plötz, 1880) & $\mathbf{\Delta}$ & 5 & A \\
\hline Bicyclus lamani (Aurivillius, 1900) & Bacelar, 1958a, b & 5 & A \\
\hline Bicyclus golo (Aurivillius, 1893) & Monard, 1956 & 5 & A \\
\hline Bicyclus s. smithi (Aurivillius, 1899) & $\Delta$ & 5 & A \\
\hline Bicyclus vansoni (Condamin, 1965) & Condamin, 1965 & 5 & $\mathrm{C}$ \\
\hline
\end{tabular}




\begin{tabular}{|c|c|c|c|}
\hline Taxon & First reference for Angola & V & $\mathrm{H}$ \\
\hline Bicyclus buea (Strand, 1912) & Larsen, 2005 & 5 & A \\
\hline Bicyclus sanaos (Hewitson, 1866) & Druce, 1875 & 5 & A \\
\hline $\begin{array}{l}\text { Hallelesis asochis congoensis (Joicey \& } \\
\text { Talbot, 1921) }\end{array}$ & Druce, 1875 , as asochis & 6 & $\mathrm{E}$ \\
\hline Brakefieldia angolensis (Kielland, 1994) & Kielland, $1994(*)$ & 1,6 & $\mathrm{C}$ \\
\hline Brakefieldia p. phaea (Karsch, 1894) & Kielland, 1994 & 5 & $\mathrm{C}$ \\
\hline Brakefieldia simonsii (Butler, 1877) & Gardiner, 2004 & 5 & $\mathrm{D}, \mathrm{F}$ \\
\hline Brakefieldia centralis (Aurivillius, 1903) & Ackery et al., 1995 & 3 & $\mathrm{C}$ \\
\hline Brakefieldia ochracea (Lathy, 1906) & Lathy, $1906(*)$ & 1,5 & $\mathrm{C}$ \\
\hline Brakefieldia eliasis (Hewitson, 1866) & Druce, 1875 & 3,5 & $\mathrm{~A}, \mathrm{~B}$ \\
\hline Mashuna upemba (Overlaet, 1955) & - & 5 & $\mathrm{E}$ \\
\hline Ypthima a. asterope (Klug, 1832) & Druce, 1875 & 5 & $\mathrm{D}$ \\
\hline $\begin{array}{l}\text { Ypthima asterope hereroica (van Son, } \\
\text { 1955) }\end{array}$ & Gardiner, 2004 & 6 & $\mathrm{D}$ \\
\hline Ypthima c. condamini (Kielland, 1982) & Larsen, 2005 & 6 & $\mathrm{C}, \mathrm{D}$ \\
\hline Ypthima granulosa (Butler, 1883) & Ladeiro, 1956 & 6 & C?,D \\
\hline Ypthima recta (Overlaet, 1955) & Kielland, 1982 & 6 & A \\
\hline Ypthima doleta (Kyrby, 1880) & Aurivillius, 1928 & 5 & A,B \\
\hline $\begin{array}{l}\text { Ypthima i. impura (Elwes \& Edwards, } \\
\text { 1893) }\end{array}$ & Aurivillius, 1928 & 5 & $\mathrm{~A}, \mathrm{D}$ \\
\hline $\begin{array}{l}\text { Ypthima impura paupera (Ungemach, } \\
\text { 1932) }\end{array}$ & Gardiner, 2004 & 6 & A,D \\
\hline Ypthima praestans (Overlaet, 1954) & $\Delta$ & 5 & A \\
\hline Ypthima pulchra (Overlaet, 1954) & $\Delta$ & 5 & A \\
\hline Ypthima diplommata (Overlaet, 1954) & Kielland, 1982 & 3,6 & $\begin{array}{l}\text { New } \\
\text { data }\end{array}$ \\
\hline Ypthimomorpha itonia (Hewitson, 1865) & Aurivillius, 1928 & 5 & $\mathrm{D}, \mathrm{E}$ \\
\hline Neita bikuarica (M \& BS, 2006) & M \& BS, $2006(*)$ & 1,5 & $\mathrm{C}$ \\
\hline Neocoenyra cooksoni (Druce, 1907$)$ & $\Delta$ & 6 & $\mathrm{C}$ \\
\hline $\begin{array}{l}\text { Mashunoides carneiromendesi (M \& BS, } \\
\text { 2009) }\end{array}$ & M \& BS, 2009 (*) & 1,5 & $\mathrm{D}$ \\
\hline \multicolumn{4}{|l|}{ NYMPHALIDAE | Charaxinae } \\
\hline $\begin{array}{l}\text { Charaxes fulvescens rubenarturi (BS \& } \\
\text { M, 2017) }\end{array}$ & BS et al., $2017(*)$ & 1,5 & A \\
\hline $\begin{array}{l}\text { Charaxes varanes vologeses (Mabille, } \\
\text { 1876) }\end{array}$ & Mabille, 1876, sub Palla (*) & 5 & $\mathrm{~B}, \mathrm{D}$ \\
\hline Charaxes candiope (Godart, 1824) & Druce, 1875 & 5 & $\mathrm{~B}, \mathrm{D}$ \\
\hline $\begin{array}{l}\text { Charaxes cynthia kinduana (Le Cerf, } \\
\text { 1923) }\end{array}$ & Aurivillius, 1928 & 5 & $\mathrm{~A}, \mathrm{D}$ \\
\hline Charaxes macclounii (Butler, 1895) & van Someren, 1970 & 6 & A \\
\hline Charaxes macclouni carvalhoi (BS, 1983) & BS, $1983(*)$ & 1,5 & A \\
\hline $\begin{array}{l}\text { Charaxes protoclea protonothodes (van } \\
\text { Someren, 1971) }\end{array}$ & Aurivillius, 1928, as protoclea & 5 & $\mathrm{~A}, \mathrm{~B}$ \\
\hline Charaxes lucretius saldanhai (BS, 1983) & BS, $1983(*)$ & 1,5 & A \\
\hline
\end{tabular}




\begin{tabular}{|c|c|c|c|}
\hline Taxon & First reference for Angola & $\mathrm{V}$ & $\mathrm{H}$ \\
\hline $\begin{array}{l}\text { Charaxes brutus angustus (Rothschild, } \\
\text { 1900) }\end{array}$ & Druce, 1875 , as brutus & 5 & $\mathrm{~A}, \mathrm{~B}, \mathrm{D}$ \\
\hline $\begin{array}{l}\text { Charaxes brutus natalensis (Staudinger, } \\
1885 \text { ) }\end{array}$ & van Someren, 1970 & 6 & $\mathrm{~A}, \mathrm{~B}, \mathrm{D}$ \\
\hline Charaxes c. castor $($ Cramer, 1775$)$ & Druce, 1875 & 5 & $\mathrm{~A}, \mathrm{~B}$ \\
\hline $\begin{array}{l}\text { Charaxes druceanus proximans (Joicey \& } \\
\text { Talbot, 1922) }\end{array}$ & Aurivillius, 1928 & 5 & A,D \\
\hline $\begin{array}{l}\text { Charaxes eudoxus mechowi (Rothschild, } \\
\text { 1900) }\end{array}$ & Rothschild, $1900(*)$ & 5 & A,D \\
\hline $\begin{array}{l}\text { Charaxes eudoxus mitchelli (Plantrou \& } \\
\text { Howarth, 1977) }\end{array}$ & $\boldsymbol{0}$ & 5 & A,D \\
\hline Charaxes s. saturnus (Butler, 1866) & $\begin{array}{l}\text { Druce, } 1875 \text {, as } C \text {. pelias } \\
\text { brunnescens }\end{array}$ & 5 & $\mathrm{~A}, \mathrm{D}$ \\
\hline Charaxes p. pollux (Cramer, 1775) & Druce, 1875 & 5 & $\mathrm{~A}, \mathrm{C}$ \\
\hline $\begin{array}{l}\text { Charaxes numenes aequatorialis (van } \\
\text { Someren, 1972) }\end{array}$ & Aurivillius, 1928 , as numenes & 5 & A \\
\hline $\begin{array}{l}\text { Charaxes tiridates tiridatinus (Röber, } \\
\text { 1936) }\end{array}$ & Druce, 1875 , the species & 5 & A \\
\hline $\begin{array}{l}\text { Charaxes ameliae amelina (Joicey \& } \\
\text { Talbot, 1925) }\end{array}$ & $\boldsymbol{\square}$ & 5 & $\mathrm{C}$ \\
\hline $\begin{array}{l}\text { Charaxes b. bohemani (Felder \& Felder, } \\
1859 \text { ) }\end{array}$ & Druce, 1875 & 5 & $\mathrm{D}$ \\
\hline Charaxes p. pythodoris (Hewitson, 1873) & Hewitson, $1873(*)$ & 5 & $\mathrm{~B}$ \\
\hline $\begin{array}{l}\text { Charaxes smaragdalis leopoldi } \\
\text { (Ghesquiére, 1933) }\end{array}$ & van Someren, 1964 & 2 & A \\
\hline Charaxes zingha $($ Stoll, 1780) & Bacelar, 1958a, b & 5 & A \\
\hline $\begin{array}{l}\text { Charaxes a. achaemenes (Felder \& } \\
\text { Felder, 1867) }\end{array}$ & van Someren, 1970 & 5 & $\mathrm{C}, \mathrm{D}$ \\
\hline Charaxes e. etesipe (Godart, 1824) & & 5 & A,E \\
\hline Charaxes p. penricei (Rothschild, 1900) & Rothschild, $1900(*)$ & 5 & C,D \\
\hline $\begin{array}{l}\text { Charaxes penricei dealbata (van } \\
\text { Someren, 1966) }\end{array}$ & van Someren, $1966(*)$ & 2,5 & A \\
\hline $\begin{array}{l}\text { Charaxes jahlusa angolensis (M \& BS, } \\
\text { 2017) }\end{array}$ & BS et al., $2017(*)$ & 1,5 & $\mathrm{~A}$ \\
\hline $\begin{array}{l}\text { Charaxes eupale latimargo (Joicey \& } \\
\text { Talbot, 1921) }\end{array}$ & Druce, 1875 as eupale & 5 & A \\
\hline Charaxes minor karinae (Bouyer, 1999) & Bouyer, $1999(*)$ & 1,5 & A \\
\hline $\begin{array}{l}\text { Charaxes anticlea proadusta (van } \\
\text { Someren, 1971) }\end{array}$ & Aurivillius, 1928 , as anticlea & 5 & A \\
\hline Charaxes h. hildebrandti (Dewitz, 1879) & Dewitz, $1879(*)$ & 5 & A \\
\hline $\begin{array}{l}\text { Charaxes hildebrandti katangensis } \\
\text { (Talbot, 1928) }\end{array}$ & BS \& M, 2014 & 5 & A \\
\hline Charaxes g. guderiana (Dewitz, 1879) & Dewitz, $1879(*)$ & 5 & A,D \\
\hline Charaxes brainei (van Son, 1966) & Henning, 1988 & 4 & $\mathrm{D}$ \\
\hline $\begin{array}{l}\text { Charaxes catachrous (van Someren \& } \\
\text { Jackson, 1952) }\end{array}$ & - & 5 & A \\
\hline
\end{tabular}




\begin{tabular}{|c|c|c|c|}
\hline Taxon & First reference for Angola & V & $\mathrm{H}$ \\
\hline Charaxes cedreatis (Hewitson, 1874) & Aurivillius, 1928 & 5 & $\mathrm{~A}, \mathrm{C}$ \\
\hline $\begin{array}{l}\text { Charaxes diversiforma (van Someren \& } \\
\text { Jackson, 1957) }\end{array}$ & van Someren, 1969 & 5 & A \\
\hline $\begin{array}{l}\text { Charaxes etheocles silvestris (Turlin, } \\
\text { 2011) }\end{array}$ & Druce, 1875 , as C. ephyra & 5 & $\mathrm{~A}, \mathrm{~B}$ \\
\hline Charaxes figueirai (BS \& M, 2014) & BS \& M, $2014(*)$ & 1,5 & $\mathrm{C}, \mathrm{D}$ \\
\hline Charaxes fulgurata (Aurivillius, 1899) & Aurivillius, $1899(*)$ & 5 & $\mathrm{C}$ \\
\hline Charaxes howarthi (Minig, 1976) & Henning, 1988 & 5 & $\mathrm{C}$ \\
\hline Charaxes phaeus (Hewitson, 1877) & Gardiner, 2004 & 6 & $\mathrm{C}, \mathrm{D}$ \\
\hline Charaxes variata (van Someren, 1969) & - & 3,5 & $\mathrm{C}$ \\
\hline Charaxes p. paphianus (Ward, 1871) & Aurivillius, 1928 & 5 & $\mathrm{~A}$ \\
\hline $\begin{array}{l}\text { Charaxes pleione congoensis (Plantrou, } \\
\text { 1989) }\end{array}$ & van Someren 1974, as pleione & 5 & A \\
\hline $\begin{array}{l}\text { Charaxes ehmckei (Homeyer \& Dewitz, } \\
\text { 1882) }\end{array}$ & Homeyer \& Dewitz, 1882 (*) & 1,5 & $\mathrm{~A}, \mathrm{~B}$ \\
\hline Charaxes zoolina (Westwood, 1850) & Gardiner, 2004 & 6 & A \\
\hline $\begin{array}{l}\text { Charaxes kahldeni (Homeyer \& Dewitz, } \\
\text { 1882) }\end{array}$ & Homeyer \& Dewitz, $1882(*)$ & 5 & A \\
\hline Charaxes $n$. nichetes (Grose-Smith, 1883) & Aurivillius, 1928 & 5 & A \\
\hline $\begin{array}{l}\text { Charaxes nichetes pantherinus Rousseau- } \\
\text { (Decelle, 1934) }\end{array}$ & - & 5 & A \\
\hline Charaxes lycurgus (Fabricius, 1793) & Plantrou, 1978 & 5 & $\mathrm{~A}$ \\
\hline Charaxes zelica rougeoti (Plantrou, 1978) & Plantrou, 1978 & 5 & A \\
\hline Charaxes doubledayi (Aurivillius, 1899) & Bacelar, 1956 & 5 & A \\
\hline Palla decius (Cramer, 1777) & Druce, 1875 & 5 & A \\
\hline $\begin{array}{l}\text { Palla publius centralis (van Someren, } \\
\text { 1975) }\end{array}$ & 0 & 5 & A \\
\hline $\begin{array}{l}\text { Palla ussheri hassoni (Turlin \& } \\
\text { Vingerhoedt, 2013) }\end{array}$ & Turlin \& Vingerhoedt, $2013(*)$ & 1,5 & A \\
\hline Palla violinitens coniger (Butler, 1896) & Aurivillius, 1928 & 5 & $\mathrm{~A}$ \\
\hline Charaxes c. crossleyi (Ward, 1871) & Aurivillius, 1928 & 6 & A \\
\hline $\begin{array}{l}\text { Charaxes eurinome ansellica (Butler, } \\
1870 \text { ) }\end{array}$ & Butler, $1870(*)$ & 5 & B \\
\hline Charaxes trajanus bambi BS \& M, 2007 & BS \& M, $2007(*)$ & 1,5 & A \\
\hline \multicolumn{4}{|l|}{ NYMPHALIDAE | Apaturinae } \\
\hline $\begin{array}{l}\text { Apaturopsis c. cleocharis (Hewitson, } \\
\text { 1873) }\end{array}$ & Hewitson, $1873(*)$ & 6 & A \\
\hline
\end{tabular}

NyMPhaLIDAE | Nymphalinae

\begin{tabular}{l|l|l|l}
\hline Kallimoides rumia jadyae (Fox, 1968) & Druce, 1875, as rumia & 5 & A \\
\hline Vanessula milca buechneri (Dewitz, 1887) & - & 6 & A \\
\hline Antanartia d. delius (Drury, 1782) & - & 5 & A \\
\hline Vanessa cardui (Linnaeus, 1758) & Snellen, 1882 & 5 & C,D \\
\hline Precis antilope (Feisthamel, 1850) & Monard, 1956 & 5 & C,D \\
\hline Precis a. archesia (Cramer, 1779) & Aurivillius, 1928 & 5 & C,D \\
\hline Precis c. ceryne (Boisduval, 1847) & Druce, 1875 & 5 & A,B,E \\
\hline
\end{tabular}




\begin{tabular}{|c|c|c|c|}
\hline Taxon & First reference for Angola & $\mathrm{V}$ & $\mathrm{H}$ \\
\hline Precis coelestina (Dewitz, 1879) & Dewitz, $1879(*)$ & 5 & A \\
\hline Precis octavia sesamus (Trimen, 1883) & Druce, 1875 , as & 5 & $\mathrm{C}$ \\
\hline Precis pelarga (Fabricius, 1775) & Ladeiro, 1956 & 5 & $\mathrm{~A}, \mathrm{C}$ \\
\hline Precis actia (Distant, 1880$)$ & Aurivillius, 1928 & 5 & $\mathrm{~A}, \mathrm{C}$ \\
\hline Precis s. sinuata (Plötz, 1880) & Bacelar, 1956 & 5 & $\mathrm{~A}, \mathrm{C}$ \\
\hline Precis rauana silvicola (Schultze, 1916) & - & 5 & A \\
\hline $\begin{array}{l}\text { Precis larseni manuscript name - (M } \\
\text { et al., 2018) }\end{array}$ & M et al., $2018(*)$ & 1,5 & $\mathrm{~A}, \mathrm{C}$ \\
\hline $\begin{array}{l}\text { Hypolimnas a. anthedon (Doubleday, } \\
\text { 1845) }\end{array}$ & Druce, 1875 , sub Diadema & 5 & $\mathrm{~A}, \mathrm{C}$ \\
\hline Hypolimnas misippus (Linnaeus, 1764) & Druce, 1875 & 5 & $\mathrm{H}$ \\
\hline Hypolimnas d. dinarcha (Hewitson, 1865) & Bacelar, 1958a, b & 5 & A \\
\hline Hypolimnas m. monteironis (Druce, 1874) & Druce, $1874(*)$ & 5 & A \\
\hline Hypolimnas s. salmacis (Drury, 1773) & Druce, 1875 & 5 & A \\
\hline Salamis c. cacta (Fabricius, 1793) & Ackery et al., 1995 & 5 & A \\
\hline $\begin{array}{l}\text { Protogoniomorpha anacardii ansorgei } \\
\text { (Rothschild, 1904) }\end{array}$ & Rothschild, $1904(*)$ & 5 & $\mathrm{~A}, \mathrm{C}$ \\
\hline $\begin{array}{l}\text { Protogoniomorpha parhassus (Drury, } \\
\text { 1782) }\end{array}$ & Druce, 1875 , as Diadema salamis & 5 & $\mathrm{~A}, \mathrm{C}$ \\
\hline $\begin{array}{l}\text { Protogoniomorpha t. temora (Felder \& } \\
\text { Felder, 1867) }\end{array}$ & Aurivillius, 1928 & 6 & A \\
\hline Junonia artaxia (Hewitson, 1864) & Aurivillius, 1928 & 5 & $\mathrm{C}, \mathrm{D}$ \\
\hline Junonia hierta crebrene (Trimen, 1870) & Butler, 1871 & 5 & A,C,D \\
\hline $\begin{array}{l}\text { Junonia } n \text {. natalica (Felder \& Felder, } \\
\text { 1860) }\end{array}$ & Gardiner, 2004 & 6 & $\mathrm{D}$ \\
\hline $\begin{array}{l}\text { Junonia natalica angolensis (Rothschild, } \\
\text { 1918) }\end{array}$ & Rothschild, $1918(*)$ & 3,5 & $\mathrm{~A}, \mathrm{C}$ \\
\hline Junonia o. oenone (Linnaeus, 1758) & Butler, 1871 & 5 & $\mathrm{H}$ \\
\hline $\begin{array}{l}\text { Junonia orythia madagascariensis } \\
\text { (Guenée, 1865) }\end{array}$ & Bacelar, 1948 & 5 & A,C,D \\
\hline Junonia sophia infracta (Butler, 1888) & Bacelar, 1948 & 5 & A \\
\hline Junonia stygia (Aurivillius, 1894) & Aurivillius, 1928 & 5 & A \\
\hline $\begin{array}{l}\text { Junonia w. westermanni (Westwood, } \\
\text { 1870) }\end{array}$ & Hewitson, 1873 & 5 & A \\
\hline Junonia terea elgiva (Hewitson, 1864) & Aurivillius, 1928 & 5 & A \\
\hline Junonia ansorgei (Rothschild, 1899) & - & 5 & A \\
\hline $\begin{array}{l}\text { Junonia cymodoce lugens (Schultze, } \\
\text { 1912) }\end{array}$ & Aurivillius, 1909 , as cymodoce & 5 & A \\
\hline Catacroptera c. cloanthe (Stoll, 1781) & Butler, 1871 & 5 & $\mathrm{~A}, \mathrm{C}, \mathrm{D}$ \\
\hline \multicolumn{4}{|l|}{ NyMPHALIDAE | Cyrestinae } \\
\hline Cyrestis c. camillus (Fabricius, 1781) & Ladeiro, 1956 & 5 & A \\
\hline \multicolumn{4}{|l|}{ NYMPHALIDAE | Biblidinae } \\
\hline $\begin{array}{l}\text { Biblia anvatara crameri (Aurivillius, } \\
\text { 1894) }\end{array}$ & Bacelar, 1948 & 5 & $\mathrm{C}, \mathrm{D}$ \\
\hline Byblia ilithyia (Drury, 1773) & Snellen, 1882 & 5 & A,D \\
\hline
\end{tabular}




\begin{tabular}{|c|c|c|c|}
\hline Taxon & First reference for Angola & $\mathrm{V}$ & $\mathrm{H}$ \\
\hline $\begin{array}{l}\text { Mesoxantha ethosea ethoseoides (Rebel, } \\
\text { 1914) }\end{array}$ & Aurivillius, 1928 & 5 & A \\
\hline $\begin{array}{l}\text { Ariadne albifascia (Joicey \& Talbot, } \\
\text { 1921) }\end{array}$ & $\Delta$ & 5 & A \\
\hline $\begin{array}{l}\text { Ariadne enotrea archeri (Carcasson, } \\
\text { 1958) }\end{array}$ & Carcasson, $1958(*)$ & 5 & A \\
\hline $\begin{array}{l}\text { Neptidiopsis ophione nucleata (Grünberg, } \\
\text { 1911) }\end{array}$ & Aurivillius, 1928 & 5 & A \\
\hline $\begin{array}{l}\text { Eurytela dryope angulata (Aurivillius, } \\
\text { 1899) }\end{array}$ & Butler, 1871 , as dryope & 5 & A,C,D \\
\hline Eurytela h. hiarbas (Drury, 1782) & Druce, 1875 & 5 & A \\
\hline $\begin{array}{l}\text { Sevenia boisduvali omissa (Rothschild, } \\
\text { 1918) }\end{array}$ & $\Delta$ & 5 & A \\
\hline $\begin{array}{l}\text { Sevenia occidentalium penricei } \\
\text { (Rothschild \& Jordan, 1903) }\end{array}$ & Rothschild \& Jordan, 1903 (*) & 1,5 & A \\
\hline $\begin{array}{l}\text { Sevenia consors (Rothschild \& Jordan, } \\
\text { 1903) }\end{array}$ & Rothschild \& Jordan, 1903 (*) & 5 & $\mathrm{C}$ \\
\hline Sevenia t. trimeni (Aurivillius, 1899) & $\begin{array}{l}\text { Aurivillius, } 1899 \text {, as Crenis } \\
\text { natalensis var. trimeni }(*)\end{array}$ & 5 & $\mathrm{~A}, \mathrm{C}$ \\
\hline Sevenia umbrina (Karsch, 1892) & $\Delta$ & 5 & \\
\hline $\begin{array}{l}\text { Sevenia amulia intermedia (Carcasson, } \\
\text { 1961) }\end{array}$ & Aurivillius, 1928, as amulia & 5 & A,C,E \\
\hline Sevenia benguelae (Chapman, 1872) & Aurivillius, 1928 & 5 & A,C,D \\
\hline Sevenia p. pechueli (Dewitz, 1879) & Dewitz, $1879(*)$ & 5 & $\mathrm{C}$ \\
\hline \multicolumn{4}{|l|}{ NYMPHALIDAE | Limenitidinae } \\
\hline Harma theobene superna (Fox, 1968) & Fox, 1968 & 5 & A \\
\hline Cymothoe o. oemilius (Doumet, 1859) & Bacelar, 1958a, b & 5 & A \\
\hline $\begin{array}{l}\text { Cymothoe b. beckeri (Herrich-Schaeffer, } \\
1858 \text { ) }\end{array}$ & Druce, 1875 & 5 & A \\
\hline $\begin{array}{l}\text { Cymothoe haynae fumosa (Staudinger, } \\
\text { 1896) }\end{array}$ & Bacelar, 1956 & 2,5 & A \\
\hline Cymothoe confusa (Aurivillius, 1887) & Bacelar, 1956 & 5 & A \\
\hline $\begin{array}{l}\text { Cymothoe lucasii cloetensi (Seeldrayers, } \\
\text { 1896) }\end{array}$ & - & 5 & A \\
\hline Cymothoe h. harmilla (Hewitson, 1874) & Larsen, 2005 & 6 & A \\
\hline Cymothoe h. hesiodotus (Hewitson, 1869) & Aurivillius, 1928 & 6 & A \\
\hline Cymothoe h. hypatha (Hewitson, 1866) & Druce, 1875 & 5 & A \\
\hline Cymothoe lurida hesione (Weymer, 1907) & Druce, 1875 , as lurida & 5 & A \\
\hline Cymothoe altisidora (Hewitson, 1869) & Aurivillius, 1898 & 5 & A \\
\hline Cymothoe capella (Ward, 1871) & Bacelar, 1956 & 5 & A \\
\hline Cymothoe caenis (Drury, 1773) & Druce, 1874 & 5 & A \\
\hline Cymothoe jodutta ciceronis (Ward, 1871) & Bacelar, 1956 & 5 & A \\
\hline Cymothoe jodutta ehmckei (Dewitz, 1887) & • & 5 & A \\
\hline $\begin{array}{l}\text { Cymothoe cf. c. coccinata (Hewitson, } \\
\text { 1874) }\end{array}$ & - & 5 & A \\
\hline
\end{tabular}




\begin{tabular}{|c|c|c|c|}
\hline Taxon & First reference for Angola & V & $\mathrm{H}$ \\
\hline $\begin{array}{l}\text { Cymothoe excelsa deltoides (Overlaet, } \\
1944 \text { ) }\end{array}$ & D’Abrera, 1980 & 3,5 & A \\
\hline Cymothoe s. sangaris (Godart, 1824) & Druce, 1875 & 5 & A \\
\hline $\begin{array}{l}\text { Pseudoneptis bugandensis ianthe } \\
\text { (Hemming, 1964) }\end{array}$ & Snellen, 1882 , as bugandensis & 5 & A \\
\hline $\begin{array}{l}\text { Pseudacraea eurytus eurytus (Linnaeus, } \\
1758 \text { ) }\end{array}$ & Druce, 1875 & 5 & A \\
\hline $\begin{array}{l}\text { Pseudacraea d. dolomena (Hewitson, } \\
1865 \text { ) }\end{array}$ & Aurivillius, 1928 & 6 & A \\
\hline $\begin{array}{l}\text { Pseudacraea b. boisduvalii (Doubleday, } \\
1845 \text { ) }\end{array}$ & Druce, 1875 & 5 & A \\
\hline $\begin{array}{l}\text { Pseudacraea kuenowii gottbergi (Dewitz, } \\
1884 \text { ) }\end{array}$ & Williams, 2007, as kuenowii & 6 & A \\
\hline $\begin{array}{l}\text { Pseudacraea lucretia protracta (Butler, } \\
1874 \text { ) }\end{array}$ & Butler, 1874 (*) & 5 & A \\
\hline Pseudacraea poggei (Dewitz, 1879) & Gardiner, 2004 & 5 & $\mathrm{~A}, \mathrm{D}$ \\
\hline Pseudacraea semire (Cramer, 1779) & Druce, 1875 & 5 & A \\
\hline Neptis saclava marpessa (Hopffer, 1855) & Druce, 1875 & 5 & $\mathrm{~A}, \mathrm{~B}, \mathrm{C}$ \\
\hline Neptis nemetes margueriteae (Fox, 1968) & Butler, 1871 , the species & 6 & $\mathrm{C}, \mathrm{D}$ \\
\hline Neptis gratiosa (Overlaet, 1955) & $\Delta$ & 5 & $\mathrm{C}$ \\
\hline Neptis jordani (Neave, 1910$)$ & Gardiner, 2004 & 6 & $\mathrm{D}$ \\
\hline Neptis kiriakoffi (Overlaet, 1955) & - & 5 & $\mathrm{~A}, \mathrm{C}$ \\
\hline Neptis laeta (Overlaet, 1955) & Gardiner, 2004 & 5 & $\mathrm{C}$ \\
\hline Neptis morosa (Overlaet, 1955) & Larsen, 2005 & 5 & $\mathrm{~A}, \mathrm{C}$ \\
\hline Neptis s. serena (Overlaet, 1955) & Gardiner, 2004 & 5 & $\mathrm{~A}, \mathrm{C}, \mathrm{E}$ \\
\hline Neptis alta (Overlaet, 1955) & Gardiner, 2004 & 5 & $\mathrm{C}$ \\
\hline $\begin{array}{l}\text { Neptis constantiae kaumba (Condamin, } \\
\text { 1966) }\end{array}$ & - & 5 & A \\
\hline Neptis nysiades (Hewitson, 1868) & Aurivillius, 1928 & 5 & A \\
\hline Neptis nicomedes (Hewitson, 1874) & Hewitson, $1874(*)$ & 5 & A \\
\hline Neptis quintilla (Mabille, 1890) & Larsen, 2005 & 5 & A \\
\hline Neptis a. agouale (Pierre-Baltus, 1978) & - & 5 & A \\
\hline Neptis melicerta (Drury, 1773) & Aurivillius, 1928 & 5 & $\mathrm{~A}, \mathrm{D}$ \\
\hline Neptis nebrodes (Hewitson, 1874) & Hewitson, $1874(*)$ & 6 & A \\
\hline Neptis nicoteles (Hewitson, 1874) & Hewitson, $1874(*)$ & 6 & A \\
\hline Neptis e. exaleuca (Karsch, 1894) & - & 5 & A \\
\hline Evena crithea (Drury, 1773) & Fox, 1968 & 5 & A \\
\hline $\begin{array}{l}\text { Evena angustatum (Felder \& Felder, } \\
\text { 1867) }\end{array}$ & - & 5 & A \\
\hline $\begin{array}{l}\text { Euryphura c. chalcis (Felder \& Felder, } \\
1860 \text { ) }\end{array}$ & Bacelar, 1956, as E. fulminea & 5 & A \\
\hline Euryphura plautilla Hewitson, 1865 & Druce, 1875 & 5 & A \\
\hline Euryphura concordia (Hopffer, 1855) & Aurivillius, 1928 & 5 & $\mathrm{C}, \mathrm{E}$ \\
\hline Hamanumida daedalus (Fabricius, 1775) & Druce, 1875 , as Aterica meleagris & 5 & C,D,F \\
\hline Pseudargynnis hegemone (Godart, 1819) & Druce, 1874 , as Aterica clorana & 5 & $\mathrm{E}$ \\
\hline
\end{tabular}




\begin{tabular}{|c|c|c|c|}
\hline Taxon & First reference for Angola & $\mathrm{V}$ & $\mathrm{H}$ \\
\hline Aterica g. extensa (Heron, 1909) & Druce, 1875 , as Aterica cupavia & 5 & $\mathrm{~A}, \mathrm{~B}, \mathrm{C}$ \\
\hline Cynandra opis bernardii (Lagnel, 1967) & Druce, 1875 , as Aterica afer & 5 & A \\
\hline Euriphene barombina (Aurivillius, 1894) & Larsen, 2005 & 5 & A \\
\hline Euriphene iris (Aurivillius, 1903) & $\Delta$ & 5 & $\mathrm{C}$ \\
\hline Euriphene plagiata (Aurivillius, 1897) & Larsen, 2005 & 6 & A \\
\hline $\begin{array}{l}\text { Euriphene saphirina trioculata (Talbot, } \\
\text { 1927) }\end{array}$ & $\Delta$ & 6 & A \\
\hline Euriphene t. tadema (Hewitson, 1866) & 口 & 5 & A \\
\hline $\begin{array}{l}\text { Euriphene gambiae gabonica (Bernardi, } \\
\text { 1966) }\end{array}$ & Bacelar, 1958a, b & 6 & A \\
\hline $\begin{array}{l}\text { Bebearia phantasia concolor (Hecq, } \\
\text { 1988) }\end{array}$ & Druce, 1875 & 6 & A \\
\hline Bebearia languida (Schultze, 1920) & $\Delta$ & 5 & A \\
\hline Bebearia a. absolon (Fabricius, 1793) & Bacelar, 1958a, b & 5 & A \\
\hline Bebearia micans (Aurivillius, 1899) & - & 5 & A \\
\hline Bebearia zonara (Butler, 1871) & - & 5 & A \\
\hline Bebearia oxione squalida (Talbot, 1928) & Aurivillius, 1909 , as oxione & & A \\
\hline $\begin{array}{l}\text { Bebearia cocalia katera (van Someren, } \\
1939 \text { ) }\end{array}$ & - & 5 & A \\
\hline $\begin{array}{l}\text { Bebearia guineensis (Felder \& Felder, } \\
\text { 1867) }\end{array}$ & Holmes, 2001 & 6 & A \\
\hline $\begin{array}{l}\text { Bebearia sophus aruunda (Overlaet, } \\
\text { 1955) }\end{array}$ & Druce, 1875 , as sophus & 5 & A \\
\hline Bebearia plistonax (Hewitson, 1874) & Hewitson, $1874(*)$ & 5 & $\mathrm{~F}$ \\
\hline Bebearia hassoni (Hecq, 1998) & Hecq, $1998(*)$ & 1 & A \\
\hline Euphaedra medon celestis (Hecq, 1986) & Butler, 1871 , as medon & 5 & A \\
\hline Euphaedra z. zaddachii (Dewitz, 1879) & Dewitz, $1879(*)$ & 5 & A \\
\hline Euphaedra cf. sinuosa (Hecq, 1974) & - & 6 & A \\
\hline Euphaedra diffusa diffusa (Gaede, 1916) & 口 & 6 & A \\
\hline Euphaedra ansorgei (Rothschild, 1918) & - & 5 & A \\
\hline Euphaedra p. permixtum (Butler, 1873) & Bacelar, 1956 & 5 & A \\
\hline $\begin{array}{l}\text { Euphaedra divoides (BS \& M, 2018) } \\
\text { (Manuscript name) }\end{array}$ & $\begin{array}{l}\text { Staudinger, } 1886 \text {, as E. themis var. } \\
\text { innocentia (?) }\end{array}$ & 1,5 & A \\
\hline $\begin{array}{l}\text { Euphaedra adonina spectacularis (Hecq, } \\
\text { 1997) }\end{array}$ & Bacelar, 1956 & 5 & A \\
\hline Euphaedra ceres electra (Hecq, 1983) & Butler, 1871 , as ceres & 5 & A \\
\hline Euphaedra fontainei (Hecq, 1977) & $\Delta$ & 5 & A \\
\hline Euphaedra v. viridicaerulea (Bartel, 1905) & - & 5 & A \\
\hline $\begin{array}{l}\text { Euphaedra preussiana robusta (Hecq, } \\
\text { 1983) }\end{array}$ & 口 & 5 & A \\
\hline Euphaedra rezia (Hewitson, 1866) & Bacelar, 1956 & 5 & A \\
\hline Euphaedra albofasciata (Berger, 1981) & - & 5 & A \\
\hline Euphaedra disjuncta virens (Hecq, 1984) & 0 & 5 & A \\
\hline Euphaedra mayumbensis (Hecq, 1984) & $\boldsymbol{0}$ & 5 & A \\
\hline
\end{tabular}




\begin{tabular}{|c|c|c|c|}
\hline Taxon & First reference for Angola & $\mathrm{V}$ & $\mathrm{H}$ \\
\hline Euphaedra p. preussi (Staudinger, 1891) & Aurivillius, 1928 & 6 & A \\
\hline Euphaedra uigensis (BS \& M, 2017) & BS \& M, 2017 (*) & 1,5 & A \\
\hline Euphaedra c. castanoides (Hecq, 1985) & - & 5 & $\mathrm{~A}, \mathrm{~F}$ \\
\hline Euphaedra coprates (Druce, 1875) & Druce, $1875(*)$ & 5 & A \\
\hline Euphaedra e. eleus (Drury, 1782) & Aurivillius, 1928 & 5 & A \\
\hline Euphaedra simplex (Hecq, 1978) & - & 5 & A \\
\hline Euphaedra ruspina (Hewitson, 1865) & Druce, 1875 & 5 & $\mathrm{~A}, \mathrm{C}$ \\
\hline $\begin{array}{l}\text { Euphaedra harpalyce comminuera (Hecq, } \\
\text { 1999) }\end{array}$ & Hecq, $1999(*)$ & 1.5 & A \\
\hline $\begin{array}{l}\text { Euphaedra harpalyce spatiosa (Mabille, } \\
\text { 1876) }\end{array}$ & Mabille, $1876(*)$ & 6 & A \\
\hline Euphaedra losinga wardi (Druce, 1874) & Druce, 1874 & 5 & A \\
\hline Euphaedra losinga limita (Hecq, 1978) & 口 & 3,5 & A \\
\hline Euptera mocquerysi (Staudinger, 1893) & Bacelar, 1956, as E. falsathyma & 5 & A \\
\hline \multicolumn{4}{|l|}{ NYMPHALIDAE | Heliconiinae } \\
\hline Acraea r. rogersi (Hewitson, 1873) & Hewitson, $1873(*)$ & 5 & $\mathrm{~B}, \mathrm{D}$ \\
\hline Acraea e. egina (Cramer, 1775) & Druce, 1875 & 5 & $\mathrm{~A}, \mathrm{C}$ \\
\hline Acraea acrita ambigua (Trimen, 1891) & Weymer, 1901, as A. bella & 6 & $\mathrm{C}, \mathrm{D}$ \\
\hline Acraea bellona (Weymer, 1908) & Weymer, $1908(*)$ & 1,5 & $\mathrm{C}$ \\
\hline Acraea periphanes (Oberthür, 1893) & Le Doux, 1923 & 5 & $\mathrm{C}, \mathrm{E}$ \\
\hline Acraea asema (Hewitson, 1877) & Trimen, 1891 & 6 & $\mathrm{C}$ \\
\hline Acraea o. omrora $($ Trimen, 1894$)$ & Trimen, $1894(*)$ & 1,6 & $\mathrm{C}$ \\
\hline $\begin{array}{l}\text { Acraea violarum anchietai (M \& BS, } \\
\text { 2017) }\end{array}$ & M \& BS, 2017 (*) & 1,5 & C,D \\
\hline Acraea buettneri (Rogenhofer, 1890) & Trimen, 1891, as A. felina & 5 & $\mathrm{~A}, \mathrm{C}$ \\
\hline Acraea cepheus (Linnaeus, 1758) & Eltringham, 1912 & 5 & $\mathrm{~A}, \mathrm{C}$ \\
\hline Acraea atolmis (Westwood, 1881) & Aurivillius, 1928, morph acontias & 5 & $\mathrm{C}$ \\
\hline Acraea bailundensis (Wichgraf, 1918) & Wichgraf, $1918(*)$ & 3,5 & $\mathrm{C}$ \\
\hline Acraea diogenes (Suffert, 1904) & $\Delta$ & 3,5 & $\mathrm{D}$ \\
\hline Acraea guillemei (Oberthür, 1893) & Aurivillius, 1928 & 6 & $\mathrm{C}$ \\
\hline Acraea lapidorum (Pierre, 1988) & Pierre, $1988(*)$ & 1,5 & $\mathrm{C}$ \\
\hline Acraea onerata $($ Trimen, 1891$)$ & Trimen, $1891(*)$ & 1,4 & C,D \\
\hline Acraea aglaonice (Westwood, 1881) & Gardiner, 2004 & 6 & $\mathrm{D}$ \\
\hline Acraea atergatis (Westwood, 1881) & Aurivillius, 1928 & 5 & $\mathrm{D}$ \\
\hline Acraea axina (Westwood, 1881) & Aurivillius, 1928 & 5 & $\mathrm{C}$ \\
\hline Acraea c. caldarena (Hewitson, 1877) & Gardiner, 2004 & 5 & $\mathrm{C}$ \\
\hline Acraea ella (Eltringham, 1911) & Eltringham, $1911(*)$ & 4,5 & $\mathrm{C}$ \\
\hline Acraea lygus (Druce, 1875$)$ & Druce, $1875(*)$ & 5 & C, D \\
\hline Acraea natalica (Boisduval, 1847) & Aurivillius, 1928 & 5 & $\mathrm{~A}, \mathrm{C}$ \\
\hline Acraea oncaea (Hopffer, 1855) & - & 6 & $\mathrm{C}$ \\
\hline Acraea pseudegina (Westwood, 1852) & Druce, 1875 & 5 & A \\
\hline Acraea pudorella (Aurivillius, 1899) & $\Delta$ & 5 & $\mathrm{C}$ \\
\hline Acraea stenobea (Wallengren, 1860) & Trimen, 1891 & 6 & C,D \\
\hline Acraea anemosa (Hewitson, 1865) & Aurivillius, 1928 & 5 & C,D \\
\hline
\end{tabular}




\begin{tabular}{|c|c|c|c|}
\hline Taxon & First reference for Angola & V & $\mathrm{H}$ \\
\hline Acraea p. pseudolycia (Butler, 1874) & Butler, $1874(*)$ & 3,5 & $\mathrm{~A}, \mathrm{C}$ \\
\hline $\begin{array}{l}\text { Acraea acara melanophanes (Le Cerf, } \\
\text { 1927) }\end{array}$ & Pierre \& Bernaud, 2013 & 5 & $\mathrm{D}$ \\
\hline Acraea z. zetes (Linnaeus, 1758) & Druce, 1875 & 5 & $\mathrm{C}, \mathrm{D}$ \\
\hline Acraea admatha (Hewitson, 1865) & Pierre, 1979 & 6 & A \\
\hline Acraea endoscota (Le Doux, 1928) & Larsen, 2005 & 6 & A \\
\hline Acrae l. leucographa (Ribbe, 1889) & Larsen, 2005 & 6 & A \\
\hline Acraea q. quirina (Fabricius, 1781) & $\Delta$ & 5 & A \\
\hline Acraea camaena (Drury, 1773 ) & Larsen, 2005 & 6 & $\mathrm{~B}$ \\
\hline Acraea n. neobule (Doubleday, 1843) & Druce, 1875 & 5 & A,C,D \\
\hline $\begin{array}{l}\text { Acraea eugenia ochreata (Grünberg, } \\
\text { 1910) }\end{array}$ & Larsen, 2005 & 5 & A \\
\hline Acraea brainei (Henning, 1986) & Ackery et al., 1995 & 6 & $?$ \\
\hline Acraea e. epaea (Cramer, 1779) & Aurivillius, 1928 & 5 & A \\
\hline Acraea formosa (Butler, 1874) & Butler, $1874(*)$ & 5 & $\mathrm{~A}, \mathrm{~B}$ \\
\hline Acraea l. leopoldina (Aurivillius, 1895) & $\Delta$ & 3 & A \\
\hline Acraea p. poggei (Dewitz, 1879) & Dewitz, $1879(*)$ & 6 & $\mathrm{C}$ \\
\hline $\begin{array}{l}\text { Acraea alcinoe camerunica (Aurivillius, } \\
1893 \text { ) }\end{array}$ & $\mathbf{\Delta}$ & 6 & A \\
\hline $\begin{array}{l}\text { Acraea umbra macarioides (Aurivillius, } \\
1893 \text { ) }\end{array}$ & Aurivillius, 1928 & 5 & A,B \\
\hline $\begin{array}{l}\text { Acraea consanguinea intermedia } \\
\text { (Aurivillius, 1899) }\end{array}$ & Le Doux, 1937 & 2,5 & A \\
\hline Acraea excisa (Butler, 1874) & Bacelar, 1948 & 5 & A \\
\hline $\begin{array}{l}\text { Acraea pseuderyta (Godman \& Salvin, } \\
\text { 1890) }\end{array}$ & Aurivillius, 1928 & 5 & A \\
\hline $\begin{array}{l}\text { Acraea vestalis congoensis (Le Doux, } \\
\text { 1937) }\end{array}$ & - & 5 & A \\
\hline Telchinia p. perenna (Doubleday, 1847) & Aurivillius, 1928 & 5 & A \\
\hline Telchinia p. penelope (Staudinger, 1896) & - & 5 & A \\
\hline Telchinia o. oreas $($ Sharpe, 1891) & Lathy, 1906 & 6 & $\mathrm{C}$ \\
\hline Telchinia circeis (Drury, 1782) & Larsen, 2005 & 6 & A \\
\hline $\begin{array}{l}\text { Telchinia parrhasia servona (Godart, } \\
\text { 1819) }\end{array}$ & Godart, $1819(*)$ & 6 & A \\
\hline $\begin{array}{l}\text { Telchinia peneleos pelasgia (Grose- } \\
\text { Smith, 1900) }\end{array}$ & Larsen, 2005 & 5 & $\mathrm{~A}, \mathrm{C}$ \\
\hline Telchinia p. pharsalus (Ward, 1871) & Aurivillius, 1928 & 5 & A,B \\
\hline Telchinia encedana (Pierre, 1976) & Pierre, 1976 & 6 & $\mathrm{E}$ \\
\hline Telchinia e. encedon (Linnaeus, 1758) & Druce, 1875 & 5 & A,C,D \\
\hline Telchinia alciope (Hewitson, 1852) & Bacelar, 1956 & 5 & A \\
\hline Telchinia a. aurivillii (Staudinger, 1896) & $\Delta$ & 6 & A \\
\hline Telchinia esebria (Hewitson, 1861) & Butler, 1874 & 5 & $\mathrm{~B}, \mathrm{C}$ \\
\hline Telchinia j. jodutta (Fabricius, 1793) & Druce, 1875 & 5 & A \\
\hline Telchinia lycoa (Godart, 1819) & Druce, 1875 & 5 & $\mathrm{~A}, \mathrm{C}$ \\
\hline Telchinia serena (Fabricius, 1775) & Butler, 1871 & 5 & A,C,D \\
\hline
\end{tabular}




\begin{tabular}{|c|c|c|c|}
\hline Taxon & First reference for Angola & V & $\mathrm{H}$ \\
\hline Telchinia v. ventura (Hewitson, 1877) & Monard, 1956 & 6 & E \\
\hline Telchinia acerata (Hewitson, 1874) & Snellen, 1882 & 5 & $\mathrm{~A}, \mathrm{C}$ \\
\hline Telchinia oberthueri (Butler, 1895) & Bacelar, 1948 & 6 & A \\
\hline $\begin{array}{l}\text { Telchinia sotikensis karschi (Aurivillius, } \\
\text { 1899) }\end{array}$ & Aurivillius, 1928 & 5 & A \\
\hline Telchinia b. bonasia (Fabricius, 1775) & Druce, 1875 & 5 & A,C,D \\
\hline Telchinia uvui balina (Karsch, 1892) & Larsen, 2005 & 6 & A \\
\hline Telchinia o. orestia (Hewitson, 1874) & Snellen, 1882 & 5 & A \\
\hline Telchinia p. pentapolis (Ward, 1871) & $\Delta$ & 6 & A \\
\hline Telchinia induna imduna (Trimen, 1895) & & 5 & $\mathrm{C}$ \\
\hline Telchinia r. rahira (Boisduval, 1833) & Druce, 1875 & 5 & $\mathrm{C}, \mathrm{D}, \mathrm{E}$ \\
\hline Telchnia mirifica (Lathy, 1906) & Lathy, $1906(*)$ & 3 & $\mathrm{C}$ \\
\hline Lachnoptera anticlia (Hübner, 1819) & Bacelar, 1958a, b, as L. iole & 6 & A \\
\hline Phalanta e. eurytis (Doubleday, 1847) & Bacelar, 1956 & 5 & A \\
\hline $\begin{array}{l}\text { Phalanta phalantha aethiopica } \\
\text { (Rothschild \& Jordan, 1903) }\end{array}$ & Druce, 1875 & 5 & C,D \\
\hline
\end{tabular}

\section{References}

Ackery PR, Smith CR, Vane Wright RI (1995) Carcasson's African Butterflies. An annotated catalogue of the Papilionoidea and Hesperioidea of the Afrotropical Region. CSIRO, Victoria, i-xii $+803 \mathrm{pp}$

Aduse-Poku K, Vingerhoedt E, Wahlberg N (2009) Out-of-Africa again: A phylogenetic hypothesis of the genus Charaxes (Lepidoptera: Nymphalidae) based on five genes region. Mol Phylogenet Evol 53:463-478

Aurivillius C In Seitz A (1928) Les Macrolepidopteres du Globe. Les Macrolepidoptères de la Faune Ethiopienne 13(4):615 pp + 80 pl. E. Le Moult, Paris

Bacelar A (1948) Lepidópteros de África principalmente das colónias portuguesas (colecção do Museu Bocage). Arquivos do Museu Bocage 19:165-207

Bacelar A (1956) Lepidópteros (Rhopalocera) de Buco Zau, enclave de Cabinda, Angola. Anais da Junta de Investigações do Ultramar 11(3):175-197

Bacelar A (1958a) Alguns Lepidópteros (Rhopalocera) do enclave de Cabinda. Revista portuguesa de Zoologia e Biologia Geral 1(2/3):197-217

Bacelar A (1958b) Alguns Lepidópteros (Rhopalocera) da África Ocidental portuguesa. Revista portuguesa de Zoologia e Biologia Geral 1(4):311-330

Bacelar A (1961) Lepidópteros do Bié (Rhopalocera) da colecção do Colégio de São Bento, em Luso (Angola). Memórias da Junta de Investigações do Ultramar (2)23:61-81

Berger LA (1979) Espèces peu connues et descriptions de nouvelles sous-espèces de Mylothris (Lepidoptera Pieridae). Revue de Zoologie Africaine 93(1):1-9

Bethune-Baker GT (1914) Notes on the taxonomic value of genital armature in Lepidoptera. Trans Entomol Soc London 1914:314-337

Bivar-de-Sousa A (1983) Contribuição para o conhecimento dos lepidópteros de Angola ( $3^{\text {a }}$ nota). Dados sobre a ocorrência do género Charaxes (Lep. Nymphalidae) em Angola (1 $1^{\mathrm{a}}$ parte). Actas do I Congresso Ibérico de Entomologia 1:107-119

Bivar-de-Sousa A, Fernandes JA (1964) Contribuição para o conhecimento dos Lepidópteros de Angola. Boletim da Sociedade Portuguesa de Ciências Naturais (2) 10(25):104-115

Bivar-de-Sousa A, Mendes LF (2006) On the genus Euxanthe Hübner, 1819 in Angola, with description of a new subspecies (Lepidoptera, Nymphalidae, Charaxinae). Nouvelle Revue d'Entomologie 23(4):369-376 
Bivar-de-Sousa A, Mendes LF (2007) New data on the Uranothauma from Angola, with description of a new species (Lepidoptera: Lycaenidae: Polyommatinae). Boletin Sociedad Entomológica Aragonesa 41:73-76

Bivar-de-Sousa A, Mendes LF (2009a) On a new species of the genus Princeps Hübner, (1807) from Cabinda (Angola) (Lepidoptera: Papilionidae). SHILAP, Revista de Lepidopterologia 37(147):313-318

Bivar-de-Sousa A, Mendes LF (2009b) New data on the Angolan Charaxes of the "etheocles group" with description of a new species (Lepidoptera, Nymphalidae, Charaxinae). Boletim da Sociedade portuguesa de Entomologia 8(15)(229):293-309

Bivar-de-Sousa A, Mendes LF (2014) New data on the Angolan Charaxesof the "etheocles group" with description of a new species (Lepidoptera, Nymphalidae, Charaxinae). Boletim da Sociedade portuguesa de Entomologia 229(8-15):293-309

Bivar-de-Sousa A, Mendes LF, Vasconcelos S (2017) Description of one new species and one new subspecies of Nymphalidae from Angola (Lepidoptera: Papilionoidea). SHILAP, Revista de Lepidopterologia 45(178):227-236

Bivar-de-Sousa A, Mendes LF (2018, in press) The "themis group" of Euphaedra (Euphaedrana) in Angola. Revision and description of one new species (Lepidoptera: Nymphalidae: Limenetidinae). Boletim da Sociedade portuguesa de Entomologia

Bouyer T (1999) Note sur les Charaxes du «groupe eupale» avec description d'une nouvelle sousespèce (Lepidoptera Nymphalidae). Entomologia Africana 4(1):37-40

Butler AG (1872) On a small collection of butterflies from Angola. Proc Zool Soc London 1871:721-725

Carvalho EL (1962) Alguns Papilionídeos da Lunda (Lepidoptera). Publicações Culturais da Companhia dos Diamantes de Angola 60:163-170

Condamin M (1966) Mise au point sur les Neptis au facies d' «14avann» (Lepidoptera: Nymphalidae). Bulletin de l'Institut Fondamental de l'Afrique Noire 28(A)(3):1008-10029

D'Abrera B (1980) Butterflies of the afrotropical region. Lansdowe Ed., Melbourne, pp i-xx + $1-593$

Dhungel B, Wahlberg N (2018) Molecular systematics of the subfamily Limenitidinae (Lepidoptera: Nymphalidae). PeerJ 6:e4311

Druce H (1875) A list of the collections of diurnal Lepidoptera made by J. J. Monteiro in Angola with description of some new species. Proc Zool Soc London 27:406-417

Eltringham H (1912) A monograph of the African species of the genus Acraea Fab., with a supplement of those of the Oriental Region. Trans Entomol Soc London 1912(1):1-374

Espeland M, Breinholt J, Willmott KR et al (2018) A comprehensive and dated phylogenomic analysis of butterflies. Curr Biol 28:1-9

Evans WH (1937) Catalog of African Hesperiidae (Indicating the classification and nomenclature adopted in the British Museum). British Museum, London, 212pp + $30 \mathrm{pl}$

Gardiner A (2004) Chapter 10. Butterflies of the four corners area. In: Timberlake JR, Childers SL (eds) Biodiversity of the Four Corners Area: Technical Review, vol 15. Occasional Publications on Biodiversity, Bulawayo, pp 381-397

Hecq J (1997) Euphaedra. Lambillionea \& Hecq, Tervuren \& Mont-Sur-Marchienne, 121 pp + $48 \mathrm{pl}$

Heikkilä M, Kaila L, Mutanen M et al (2012) Cretaceous origin and repeated Tertiary diversification of the redefined butterflies. Proc R Soc Lond B 279:1093-1099

Henning SF (1988) The Charaxes Butterflies of Africa. Aloe Books, Johannesburg, 457 pp

Holmes CWN (2001) A reaprisal of the Bebearia mardania complex (Lepidoptera, Nymphalidae). Trop Zool 14:31-62

Homeyer A, Dewitz H (1882) Drei neue westafrikanische Charaxes. Berliner Entomologisches Zeitschrift 26(II):381-383

JIU (1948-1963) Cartas do levantamento aerofotogramétrico de Angola (escala 1: 250,000). Junta de Investigações do Ultramar: folhas:8-471

Kielland J (1982) Revision of the genus Ypthima in the Ethiopian Region excluding Madagascar (Lepidoptera, Satyridae). Tijdschrift voor Entomologie 125(5):99-154 
Kielland J (1994) A revision of the genus Henotesia (excluding Madagascar and other Indian Ocean islands) (Lepidoptera Satyridae). Lambillionea 94(2):235-273

Ladeiro JM (1956) Lepidópteros de Angola (estudo de uma colecção oferecida ao Museu Zoológico de Coimbra). Anais da Junta de Investigações do Ultramar 11(3):151-172

Larsen TB (2005) Butterflies of West Africa. Text volume: 1-595. Apollo Books, Stenstrup

Latreille PA, Godart JB (1819) Encyclopédie Méthodologique. Histoire Naturelle (Zoologie), 9, Entomologie, Paris, i-iv + 1-328

Lautenschläger T, Neinhuis C (eds) (2014) Riquezas Naturais do Uíge - Uma breve Introdução sobre o estado atual. A utilização, a Ameaça e a Preservação da Biodiversidade. Technische Universität Dresden, Dresden, 125 pp

Libert M (1999) Revision des Epitola (ls). Révision des genres Epitola Westwood, Hypophytala Clench et Stempfferia Jackson et description de trois nouveaux genres (Lepidoptera Lycaenidae). ABRI \& Lambillionea, Nairobi/Tervuren, pp 1-219, pl. I-XVI

Libert M (2000) Révision du genre Mimacraea Butler, avec description de quatre nouvelles espèces et deux nouvelles sous-espèces (Lepidoptera, Lycaenidae). Lambillionea \& ABRI, Tervuren/ Nairobi, pp 1-73

Libert M (2004) Revision du genre Oxylides Hübner (Lepidoptera, Lycaenidae). Lambillionea 104(2):143-158

Mendes LF, Bivar-de-Sousa A (2006a) A new species of Neita van Son (Nymphalidae, Satyrinae) from southern Angola. Boletin Sociedad Entomológica Aragonesa 39:95-96

Mendes LF, Bivar-de-Sousa A (2006b) Notes and descriptions of Afrotropical Appias butterflies (Lepidoptera: Pieridae). Boletin Sociedad Entomológica Aragonesa 39:151-160

Mendes LF, Bivar-de-Sousa A (2007a) New species of Cooksonia Druce, 1905 from Angola (Lepidoptera: Lycaenidae, Lipteninae). SHILAP, Revista de Lepidopterologia 35(138):265-268

Mendes LF, Bivar-de-Sousa A (2007b) On the genus Eagris Guenée, 1863 in Angola (Lepidoptera: Hesperiidae). SHILAP, Revista de Lepidopterologia 35(139):311-316

Mendes LF, Bivar-de-Sousa A (2009a) Description of a new species of Mylothris from northern Angola (Lepidoptera Pieridae). Bolletino della Societá Entomológica Italiana 141(1):55-58

Mendes LF, Bivar-de-Sousa A (2009b) New account on the butterflies of Angola. The genus Leptomyrina (Lepidoptera Lycaenidae). Bolletino della Societá Entomológica Italiana 141(2):109-112

Mendes LF, Bivar-de-Sousa A (2009c) On a new south-eastern Angolan Satyrine butterfly belonging to a new genus (Lepidoptera, Nymphalidae). Entomologia Africana 14(2):5-8

Mendes LF, Bivar-de-Sousa A (2009d) The genus Abantis Hopffer, 1855 in Angola and description of a new species (Lepidoptera: Hesperiidae, Pyrginae). SHILAP, Revista de Lepidopterologia 37(147):313-318

Mendes LF, Bivar-de-Sousa A (2012) Notes on the species of Hypolycaena (Lepidoptera, Lycaenidae, Theclinae) known to occur in Angola. Boletin Sociedad Entomológica Aragonesa 50:193-197

Mendes LF, Bivar-de-Sousa A, Figueira R (2013a) Butterflies of Angola / Borboletas diurnas de Angola. Lepidoptera. Papilionoidea, I. Hesperiidae, Papilionidae. IICT and CIBIO, Lisboa and Porto, $288 \mathrm{pp}$

Mendes LF, Bivar-de-Sousa A, Figueira R et al (2013b) Gazetteer of the Angolan localities known for beetles (Coleoptera) and butterflies (Lepidoptera: Papilionoidea). Boletim da Sociedade Portuguesa de Entomologia 8(14/228):257-290

Mendes LF, Bivar-de-Sousa A, Vasconcelos S et al (2017) Description of two new subspecies and notes on Charaxes Ochenheimer, 1816 of Angola (Lepidoptera: Nymphalidae). SHILAP, Revista de Lepidopterologia 45(178):299-315

Mendes LF, Bivar-de-Sousa A, Vasconcelos S (2018, in press) On the butterflies of genus Precis Hübner, 1819 from Angola and description of a new species (Lepidoptera: Nymphalidae: Nymphalinae). SHILAP Revista de Lepidopterologia

Monard A (1956) Compendium Entomologicum Angolae - 1. Insecta. VI - Ord. Lepidoptera. Anais da Junta de Investigações do Ultramar 11(3):119-128 
NHM - Natural History Museum (2004). Wallowtails. Available at: http://internet.nhm.ac.uk/cgibin/perth/wallowtails/list.dsml

Pierre J (1988) Les Acraea du super-group «egina»: Révision et phylogénie (Lepidoptera : Nymphalidae). Annales de la Société Entomologique de France 24(3):263-287

Pierre J, Bernaud D (2013) Butterflies of the world. NymphalidaeXXIII. Acraea subgenus Acraea. Goecke \& Evers, Keltern, 39:1-8, pl. 1-28

Rothschild W, Jordan K (1903) Lepidoptera collected by Oscar Neumann in north-east Africa. Novitates Zoologicae 10(3):491-542

Smith CR, Vane-Wright RI (2001) A review of the Afrotropical species of the genus Graphium (Lepidoptera: Rhopalocera: Papilionidae). Bull Nat His Mus London (Entomol) 70(2):503-719

Stempffer H (1957) Les Lépidoptères de l'Afrique noire française. Lycaenidés. Initiations Africaines 14(3):1-228

Stempffer H, Bennett NH (1963) A new genus of Lipteninae (Lepidoptera: Lycaenidae). Bull Brit Mus (Nat His) (Entomol) 13:171-194

Talbot G (1944) A preliminary revision of the genus Mylothris Hübn. (1819) (Lep. Rhop. Pieridae). T Roy Ent Soc London 94(2):155-185

Tite GE (1959) New species and notes on the genus Lepidochrysops (Lepidoptera, Lycaenidae). The Entomologist 92:158-163

Tite GE (1961) New species of the genus Lepidochrysops (Lepidoptera, Lycaenidae). The Entomologist 94:21-25

Tite GE, Dickson CGC (1973) The genus Aloeides and allied genera. Bull Brit Mus (Nat His) (Entomol) 29:227-280

Trimen R (1891) On butterflies collected in tropical south-western Africa by Mr. A. W. Eriksson. Proc Zool Soc 1891:59-107

Turlin B, Vingerhoedt E (2013) Butterflies of the World, supl. 23. Les Charaxinae de la faune Afrotropicale. Les genres Palla et Euxanthe. Nymphalidae: Charaxinae: Pallini et Euxanthini. Goecke \& Evers, Keltern

Weymer G (1901) Beitrag zur Lepidopterofauna von Angola. Entomologischen Zeitschriften, Stuttgart 15(17):61-64, 65-67, 69-70

Weymer G (1908) Einige neuer Lepidopteren des Deutschen Entom. National-Museums, gesammelt von Dr. F. Cr. Wellman in Benguella. Deutsche Entomologische Zeitschrift 1908:507-413

Willis CK (2009) Amist the butterflies of southwestern Angola. Metamorphosis 20(3):74-88

Williams MC (2018) Afrotropical butterflies. Available at: http://www.lepsocafrica.org/?p=publi cations\&s=atb

Open Access This chapter is licensed under the terms of the Creative Commons Attribution 4.0 International License (http://creativecommons.org/licenses/by/4.0/), which permits use, sharing, adaptation, distribution and reproduction in any medium or format, as long as you give appropriate credit to the original author(s) and the source, provide a link to the Creative Commons licence and indicate if changes were made.

The images or other third party material in this chapter are included in the chapter's Creative Commons licence, unless indicated otherwise in a credit line to the material. If material is not included in the chapter's Creative Commons licence and your intended use is not permitted by statutory regulation or exceeds the permitted use, you will need to obtain permission directly from the copyright holder.

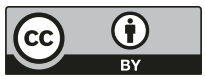

